\title{
Reconstruction of Near-Surface Tornado Wind Fields from Forest Damage
}

\author{
VERONIKA BECK* \\ Physik-Department T34, Technische Universität München, Garching, and Deutsches Zentrum für Luft- und Raumfahrt, \\ Institut für Physik der Atmosphäre, Wessling, Germany \\ NiKOLAi DOTZEK ${ }^{+}$ \\ Deutsches Zentrum für Luft- und Raumfahrt, Institut für Physik der Atmosphäre, and European Severe \\ Storms Laboratory, Wessling, Germany
}

(Manuscript received 14 April 2009, in final form 22 January 2010)

\begin{abstract}
Tornado intensity is usually inferred from the damage produced. To foster postevent tornado intensity assessments, the authors present a model to reconstruct near-surface wind fields from forest damage patterns. By comparing the structure of observed and simulated damage patterns, essential parameters to describe a tornado near-surface wind field are derived, such as the ratio $G_{\max }$ between circular and translational velocity, and the deflection angle $\alpha$ between peak wind and pressure gradient. The model consists of a wind field module following the Letzmann analytical tornado model and a tree module based on the mechanistic HWIND tree model to assess tree breakage. Using this method, the velocity components of the near-surface wind field, the track of the tornado center, and the spatial distribution of the Fujita scale along and across the damage path can be assessed. Necessary requirements to apply the model are knowledge of the tornado translation speed (e.g., from radar observations) and a detailed analysis of the forest damage patterns. One of the key findings of this analysis is that the maximum intensity of the tornado is determinable with an uncertainty of only $\left(G_{\max }+1\right)$ times the variability of the usually well-known tornado translation speed. Further, if Letzmann's model is applied and the translation speed of the tornado is known, the detailed tree model is unnecessary and could be replaced by an average critical velocity for stem breakage $v_{\text {crit }}$ independent of the tree species. Under this framework, the F3 and F2 ratings of the tornadoes in Milosovice, Czech Republic, on 30 May 2001 and Castellcir, Spain, on 18 October 2006, respectively, could be verified. For the Milosovice event, the uncertainty in peak intensity was only $\pm 6.0 \mathrm{~m} \mathrm{~s}^{-1}$. Additional information about the structure of the near-surface wind field in the tornado and several secondary vortices was also gained. Further, this model allows for distinguishing downburst damage patterns from those of tornadoes.
\end{abstract}

\section{Introduction}

Postevent assessment of wind fields in tornadoes or other small-scale damaging wind phenomena like downbursts (see Doswell 2001 for an overview) is a topic of great practical and scientific relevance. Intensity, that is, peak wind speed and subsequently the Fujita-scale rating (F scale; e.g., Fujita 1981; cf. Table 1), of such events is

\footnotetext{
* Current affiliation: Max-Planck-Institut für Biogeochemie, Jena, Germany.

+ Deceased.

Corresponding author address: Veronica Beck, Max-PlanckInstitut für Biogeochemie, Hans-Knöll-Str. 10, D-07745 Jena, Germany.

E-mail: vbeck@bgc-jena.mpg.de
}

usually inferred from site surveys or aerial photography of the damage swaths. This method is not without shortcomings (cf. Doswell and Burgess 1988; Brooks and Doswell 2001). First, the actual strength of damaged manmade structures or vegetation may only be known approximately. Second, by focusing only on peak intensity in F-scale ratings, the relative size of the area with that peak intensity compared to the total size of the damage swath remains obscure. And third, it might be impossible to determine peak intensity in the absence of suitable damage indicators. While damaged objects provide an estimate of the lower limit of wind speeds, inference of an upper limit of wind speeds requires objects strong enough to remain undamaged by the storm.

To address these shortcomings in part, the "Enhanced Fujita" (EF scale) was implemented in the United States 

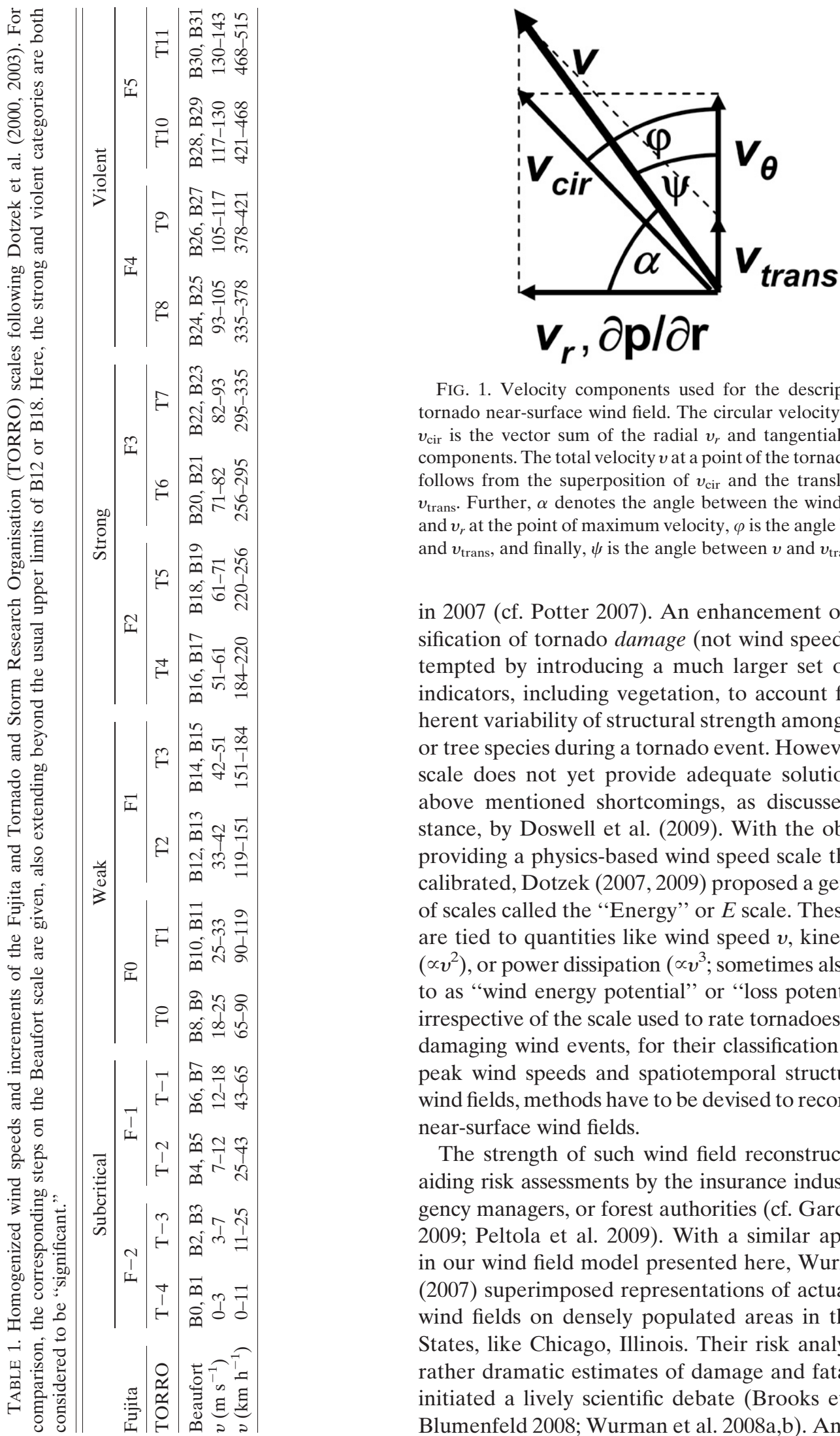

FIG. 1. Velocity components used for the description of the tornado near-surface wind field. The circular velocity component $v_{\text {cir }}$ is the vector sum of the radial $v_{r}$ and tangential $v_{\theta}$ velocity components. The total velocity $v$ at a point of the tornado wind field follows from the superposition of $v_{\text {cir }}$ and the translation speed $v_{\text {trans. }}$ Further, $\alpha$ denotes the angle between the wind direction $v$ and $v_{r}$ at the point of maximum velocity, $\varphi$ is the angle between $v_{\text {cir }}$ and $v_{\text {trans }}$, and finally, $\psi$ is the angle between $v$ and $v_{\text {trans. }}$.

in 2007 (cf. Potter 2007). An enhancement of the classification of tornado damage (not wind speeds) was attempted by introducing a much larger set of damage indicators, including vegetation, to account for the inherent variability of structural strength among buildings or tree species during a tornado event. However, the EF scale does not yet provide adequate solutions to the above mentioned shortcomings, as discussed, for instance, by Doswell et al. (2009). With the objective of providing a physics-based wind speed scale that can be calibrated, Dotzek $(2007,2009)$ proposed a generic class of scales called the "Energy" or $E$ scale. These $E$ scales are tied to quantities like wind speed $v$, kinetic energy $\left(\propto v^{2}\right)$, or power dissipation $\left(\propto v^{3}\right.$; sometimes also referred to as "wind energy potential" or "loss potential"). Yet irrespective of the scale used to rate tornadoes and other damaging wind events, for their classification regarding peak wind speeds and spatiotemporal structure of the wind fields, methods have to be devised to reconstruct the near-surface wind fields.

The strength of such wind field reconstructions is in aiding risk assessments by the insurance industry, emergency managers, or forest authorities (cf. Gardiner et al. 2009; Peltola et al. 2009). With a similar approach as in our wind field model presented here, Wurman et al. (2007) superimposed representations of actual tornado wind fields on densely populated areas in the United States, like Chicago, Illinois. Their risk analysis led to rather dramatic estimates of damage and fatalities and initiated a lively scientific debate (Brooks et al. 2008; Blumenfeld 2008; Wurman et al. 2008a,b). An approach 

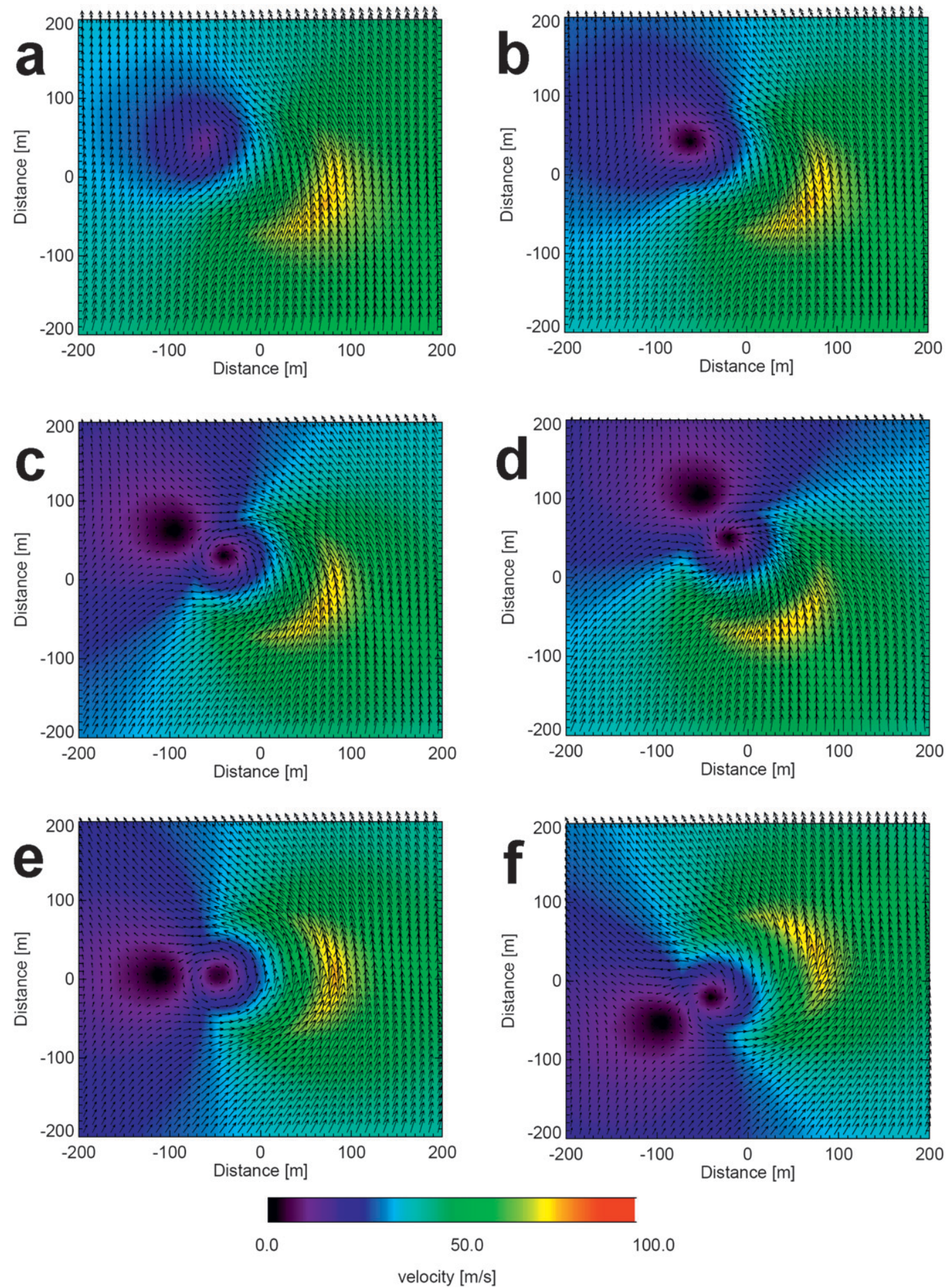

FIG. 2. The vortex field for a constant $\alpha=60^{\circ}$ and varying values of (a) $G_{\max }=0.75$, (b) $G_{\max }=1.0$, and (c) $G_{\max }=$ 1.5. The separation into the two calm points occurs at $G_{\max }=1.0$. The wind fields for a constant $G_{\max }=1.5$ and various (d) $\alpha=-30^{\circ}$, (e) $\alpha=-90^{\circ}$, and (f) $\alpha=-120^{\circ}$. The resulting change from spiral inflow into the vortex center (d) to spiral outflow of the vortex center (f) becomes apparent. In all panels, tornado propagation is from bottom to top. 

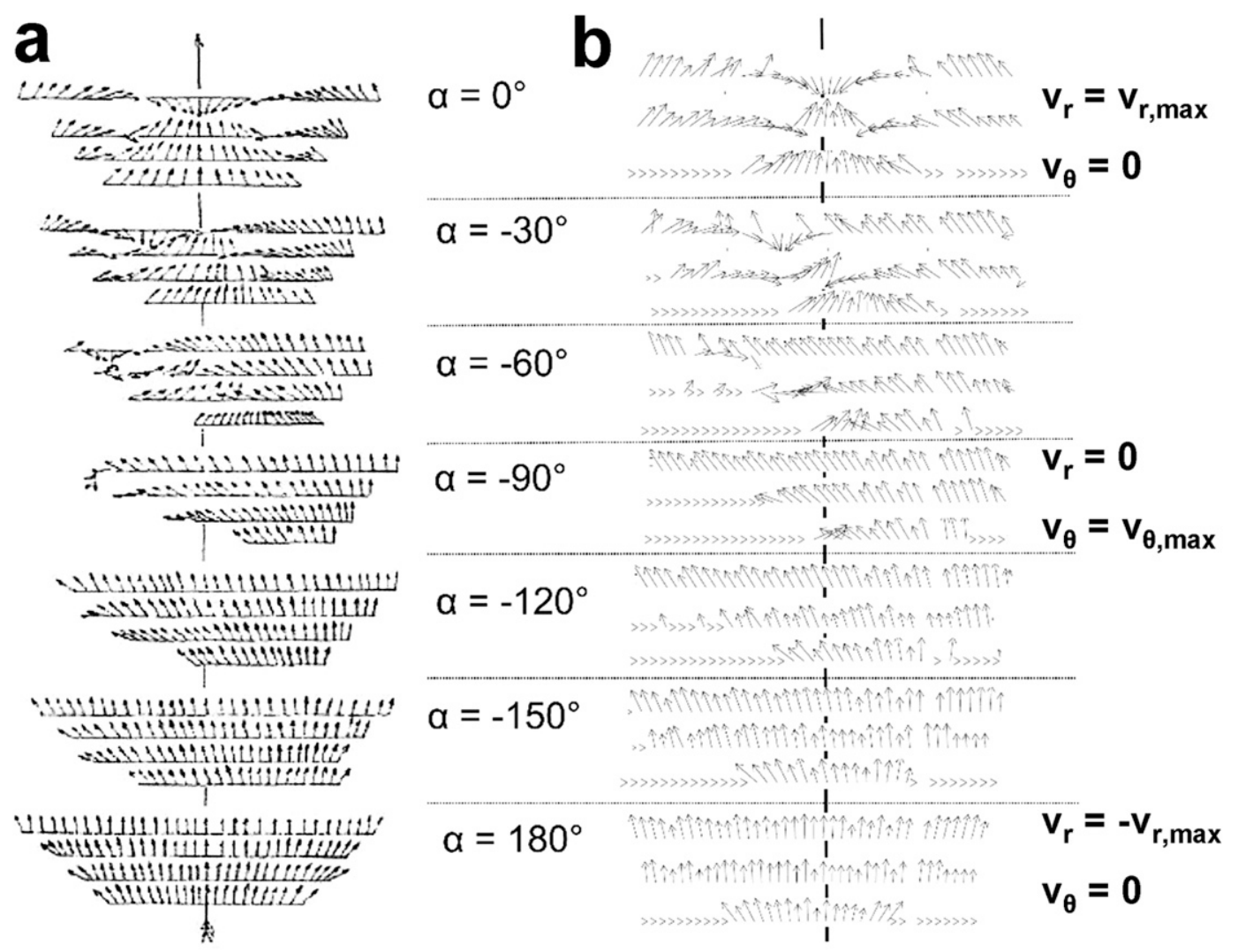

FIG. 3. (a) Analytically derived horizontal line cross sections of tree damage patterns perpendicular to the track of the tornado (Letzmann 1923) compared to (b) modeled horizontal line cross sections of tree damage patterns for $G_{\max }=2.0$ and varying $\alpha$ and $v_{\text {crit. }}$. For each $\alpha$ varying from $0^{\circ}$ to $180^{\circ}$, tree damage patterns for different $v_{\text {crit }}$ are shown. For each $\alpha$ in both (a) and (b), $v_{\text {crit }}$ increases from top to bottom. In (a), $v_{\text {crit }}$ is indicated as multiples of $v_{\text {trans }}-[1.0,1.5,2.0,2.5] \times v_{\text {trans. }}$ In (b), the middle panel of the damage patterns $\left(\alpha=-90^{\circ}\right)$ uses $v_{\text {crit }}$ from the tree module for a spruce forest, while the upper and lower panels use minimum and maximum values $\left(v_{\text {crit }}-25.0 \mathrm{~m} \mathrm{~s}^{-1}\right.$ and $\left.v_{\text {crit }}+25.0 \mathrm{~m} \mathrm{~s}^{-1}\right)$, respectively. In all panels, tornado propagation is from bottom to top.

analogous to the one for urban areas is also possible for risk analysis of potential damage to forests. Simulations of tornadoes passing over forests can be done to calculate the number of downed trees due to stem breakage or overturn. In conjunction with tornado intensity distributions (Dotzek et al. 2005), this could support the forest industry in developing adaptation concepts, for instance optimizing insurance, to minimize the financial burden from forest damage by severe local stormseither occurring as individual entities or embedded in frontal bands of synoptic-scale cyclones.

While there are advanced numerical simulations of near-surface tornado dynamics over terrain without vegetation (Lewellen and Lewellen 2007; Lewellen et al. 2008), the simulation of tornado damage in forests was recently taken up by Holland et al. (2006). They performed simulations of tornado forest damage patterns with a simple vortex model and a differentiated tree model. In their approach, they reinvented parts of much earlier, analytical work by Johannes Letzmann in the 1920s and 1930s (Dotzek et al. 2008). In Europe, tree damage had always been accounted for in tornado damage assessments (cf. Wegener 1917). Consequently, Letzmann $(1923,1925)$ developed his analytical model of tornado near-surface wind fields and proposed it also as a procedure for wind field reconstruction from forest damage patterns. In the 1930s, he further devised detailed guidelines for in situ and aerial forest damage assessments that would provide the optimal input to his analytical model (Letzmann 1939). His method to reconstruct tornado wind fields was occasionally applied in Europe to determine tornado wind field velocity components until the 1970s (Müldner 1950; Rossmann 1959; Euteneuer 1970). Yet, in general, Letzmann's achievements had already started to fall into oblivion during and soon after World War II and were only rediscovered by Peterson (1992a,b; see Dotzek et al. 2000, 2008). In the United States, forest damage patterns have been 


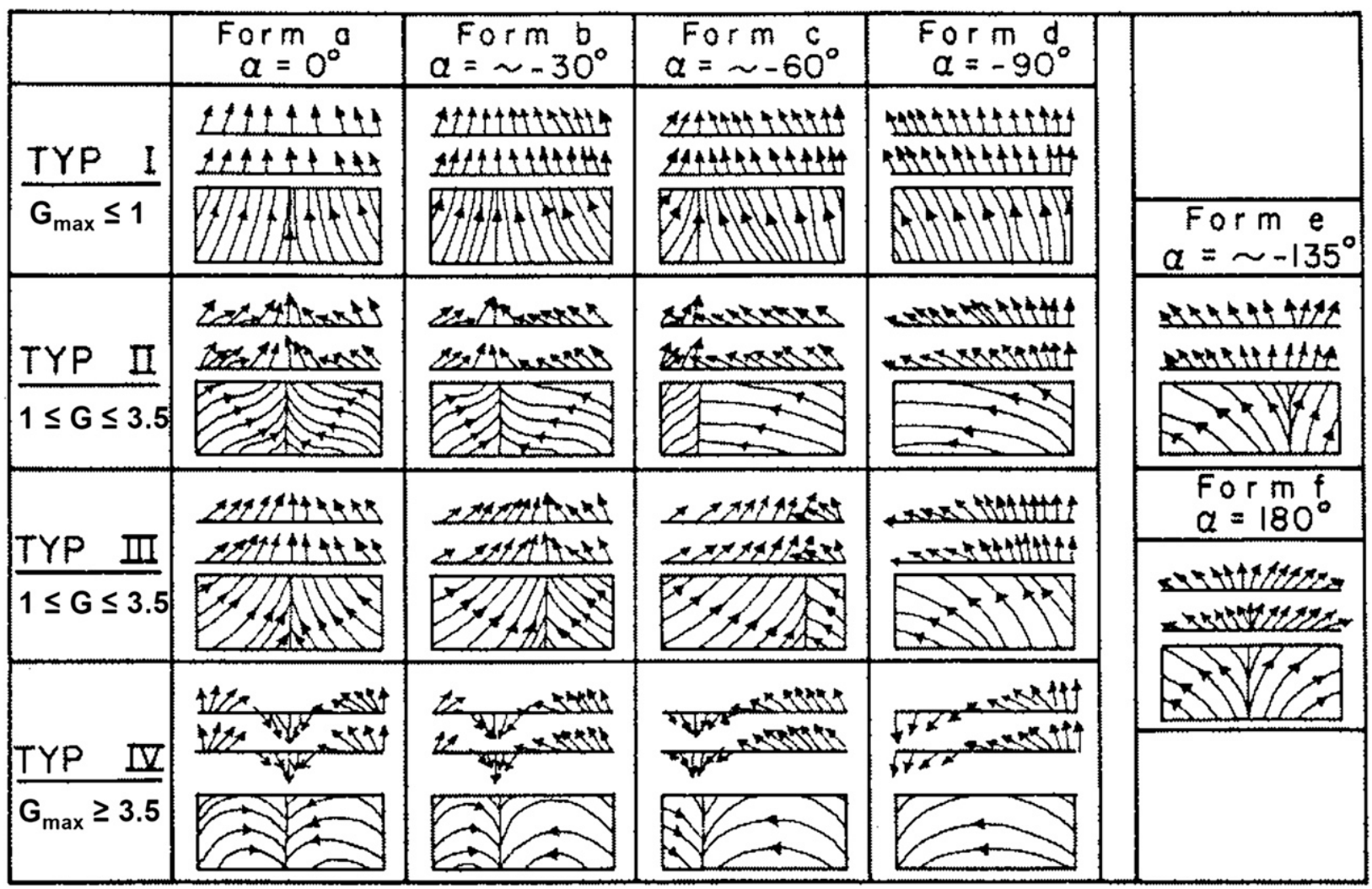

FIG. 4. Classification of the theoretical tree damage patterns into four different swath types (Letzmann 1923) for $|\alpha|<90^{\circ}$, as a function of $G_{\max }$. The damage patterns are shown for a counterclockwise rotation of the tornado vortex. For small $G_{\max }(<1.0)$, the tree with $\psi=0^{\circ}$ moves to the left for increasing values of $|\alpha|$ and no crossing of trees occurs. The difference between swath types II and III is in the higher value of $v_{\text {crit }}$ for swath type III that leads to a movement of the tree with $\psi=0^{\circ}$ to the right side of the damage pattern for increasing values of $|\alpha|$. At $|\alpha|=$ $60^{\circ}$, crossed trees can occur for both swath types. High values of $G_{\max }$ are clearly indicated by a tree with $\psi=180^{\circ}$ (swath type IV). For $|\alpha|>$ $90^{\circ}$, all tree damage patterns have a similar divergent structure. In all panels, tornado propagation is from bottom to top.

documented and analyzed in a few case studies (Hall and Brewer 1959; Budney 1965; Forbes and Wakimoto 1983; Fujita 1989; Peterson 2003). Some of these had also made brief reference to Letzmann's work, similar to Holland et al. (2006). More recently, Letzmann's work was credited by Lee and Wurman (2005) and Wurman and Alexander (2005) as well as Bech et al. (2007, 2009), who performed a simulation of the forest damage of the Castellcir, Spain, tornado, which will also serve as a case study here.

The present paper summarizes the work by Beck (2008) and is organized as follows: in section 2, the setup and validation of the model are explained. Application and verification of the method is demonstrated in section 3 via damage analysis and reconstruction of the near-surface wind fields in the tornadoes of Milosovice, Czech Republic, on 31 May 2001 and Castellcir on 18 October 2006. Section 4 provides a discussion of our results, also addressing the distinction between tornado and downburst damage patterns. Section 5 presents our conclusions.

\section{Model description and validation}

\section{a. Letzmann's analytical wind field model}

Letzmann (1923) developed a complete analytical threedimensional tornado model with a linear velocity increase in the tornado core (i.e., solid body rotation) and hyperbolical velocity decay in the tornado mantle for the tangential $v_{\theta}$ and radial $v_{r}$ velocity components of the tornado wind field (Fig. 1; cf. Beck et al. 2008). The vertical velocity component can either be constant or variable for barotropic or baroclinic vortices, respectively. For the determination of the tree damage patterns, Letzmann (1923) used a projection of the three-dimensional tornado wind field onto the horizontal $x-y$ plane. In the resulting model, $v_{\theta}$ and $v_{r}$ are described by the following relations (Letzmann 1923):

$$
v_{r, \theta}=v_{r, \theta \max }\left(\frac{r}{R_{\max }}\right)^{\gamma}, \quad r \leq R_{\max },
$$




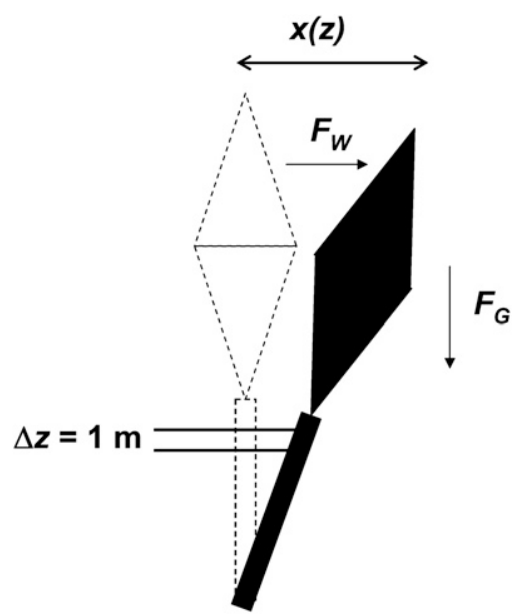

FIG. 5. Structure of $A$ of tree against the wind consisting of two isosceles triangles for the crown and a rectangle for representing the stem, which is divided into $\Delta z=1-\mathrm{m}$ segments. The arrows indicate the directions of $F_{W}, F_{G}$, and $x(z)$.

and

$$
v_{r, \theta}=v_{r, \theta \max }\left(\frac{R_{\max }}{r}\right)^{\gamma}, \quad r>R_{\max }
$$

where $R_{\max }$ indicates the radius of maximum winds, demarcating the tornado core, and $v_{r, \theta \max }$ indicates the maximum absolute value of $v_{r}$ and $v_{\theta}$ at $r=R_{\max }$, respectively. Letzmann (1923) used the exponent $\gamma$ in Eqs. (1a)-(1b) to specify the strength of the velocity increase in the tornado core and of the hyperbolical velocity decay in the tornado mantle. For $\gamma=1.0$ as often applied by Letzmann (1923), Eqs. (1a)-(1b) show exactly the velocity distribution of a Rankine vortex with a linear velocity increase in the tornado core and a hyperbolical velocity decay in the tornado mantle valid for conservation of angular momentum. By measuring tornado radial velocity profiles with the help of mobile Doppler radars, an exponent of $\gamma \approx 0.6$ was detected recently, suggesting that angular momentum might not be conserved in real tornadoes (e.g., Bluestein 2007). While $\gamma=0.6$ has been used as well, most results presented here assume $\gamma=1.0$. Additionally, there is the translation velocity $v_{\text {trans }}$ of the tornado that was assumed constant for simplicity by Letzmann (1923) and in the present work.

Contrary to other mathematical descriptions of the Rankine vortex (e.g., Kanak 2005), $v_{r}$ and $v_{\theta}$ of the Letzmann (1923) model are determined by three parameters: $v_{\text {trans }}, G_{\max }$, and $\alpha$. Here, $G_{\max }$ indicates the ratio between circular component $v_{\text {cir }}$ and $v_{\text {trans }}$ of the tornado wind field. The circular velocity component is defined as the superposition of $v_{r}$ and $v_{\theta}$. Its further superposition with $v_{\text {trans }}$ leads to the total velocity $v$ of the tornado wind field. Furthermore, $\alpha$ denotes the angle between the direction of the wind $v$ and the pressure gradient $\nabla p \propto v_{r}$ at the point of maximum velocity, where $v_{r}$ and $v_{\text {trans }}$ are perpendicular, as illustrated in Fig. 1.

The dependence of the wind field on the parameters $G_{\max }$ and $\alpha$ is shown in Fig. 2. By varying the parameter $G_{\max }$, the genesis of two calm points (Letzmann 1923) of zero total velocity, one central and one marginal point, occurs at a value of $G_{\max }=1$ (Fig. 2b). For higher values of $G_{\max }$, the distance between the two calm points increases monotonically (Fig. 2c). For the variation of $\alpha$, a change from inflow toward the vortex center (Fig. 2d) in early stages of the tornado life cycle to outflow from the vortex center (Fig. 2f) during the decaying stage is notable at a value of $|\alpha|=90^{\circ}$ (Fig. 2e).

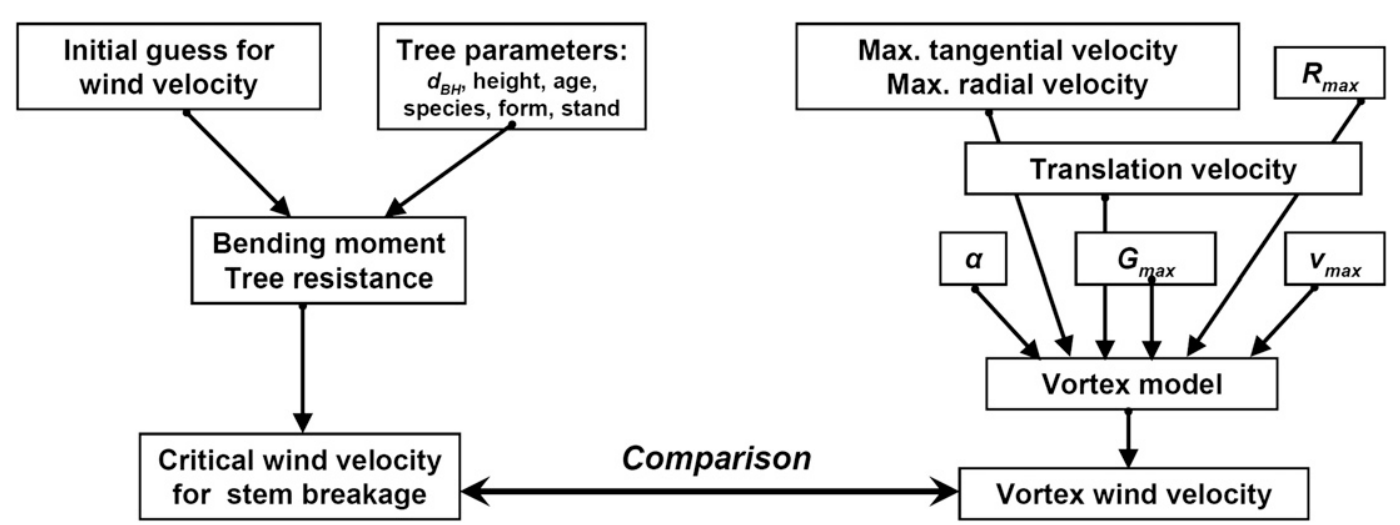

FIG. 6. Structure of the complete model consisting of a tree damage module for the calculation of $v_{\text {crit }}$ and a wind field module calculating the instantaneous wind velocity at each grid point. If the instantaneous wind velocity exceeds $v_{\text {crit }}$, the tree is considered to be broken. The structure of the model allows for the introduction of other tree damage or wind field modules in the future to calculate the specific model parameters. 

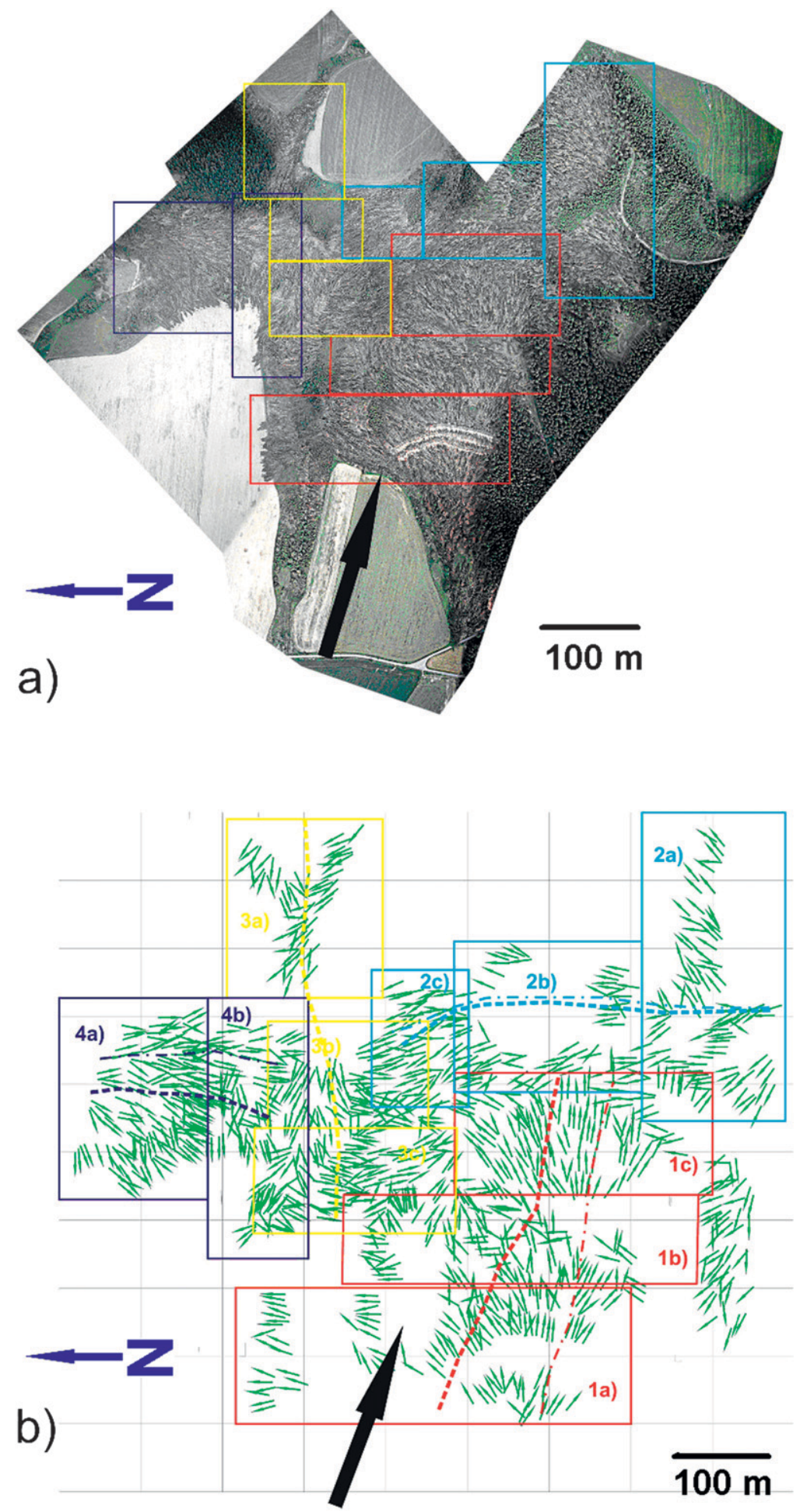

FIG. 7. (a) Aerial photograph of the forest damage produced by the Milosovice tornado (courtesy of Martin Setvák, CHMI), showing the division of the damage patterns into one main vortex (red) and three smaller vortices. (b) Digitized damage patterns containing the trace (dashed lines) as well as the divergence and convergence lines (dashed-dotted lines) of the individual vortices. In both (a) and (b), the blue arrow points north, and the black arrow indicates the propagation of the tornado toward the east-southeast. 
Tree damage patterns that depend on $G_{\max }$ and $\alpha$ have been derived by Letzmann (1923) by using the method of "individual circles" (cf. Peterson 1992a). Letzmann calculated the theoretical fall direction of the trees with respect to the direction of the translation velocity of the tornado (Fig. 3a). To characterize the tree damage patterns, a tree fall angle $\psi$ describing the deflection of downed trees from the direction of tornado translation (cf. Fig. 1) was derived by Letzmann (1923), with $\varphi$ indicating the angle between $v_{\text {cir }}$ and $v_{\text {trans }}$ :

$$
\cos \psi=\frac{v_{\text {trans }}+v_{\text {cir }} \cos \varphi}{\left(v_{\text {trans }}^{2}+v_{\text {cir }}^{2}+2 v_{\text {trans }} v_{\text {cir }} \cos \varphi\right)^{1 / 2}} .
$$

A divergence line in the vortex is always characterized by one or more downed trees with $\psi=0^{\circ}$ along a cross section perpendicular to the direction of translation. On the contrary, a convergence line is indicated by such fallen trees with either $\psi=0^{\circ}$ or $\psi=180^{\circ}$ (Letzmann 1923). Figure $3 \mathrm{a}$ demonstrates the tree damage patterns for $G_{\max }=2.0$ and varying $\alpha$ for different critical velocities for stem breakage $v_{\text {crit }}$. For the determination of $v_{\text {crit }}$, the tree model described in section $2 \mathrm{~b}$ is used.

For low $v_{\text {crit }}\left(<20.0 \mathrm{~m} \mathrm{~s}^{-1}\right.$; upper treefall pattern panels) in Fig. 3, a tree with $\psi=180^{\circ}$ occurs for $0^{\circ}<$ $|\alpha|<90^{\circ}$, while for higher $v_{\text {crit }}$ (middle and lower treefall pattern panels for each $\alpha$ ), it is replaced by a tree with $\psi=0^{\circ}$. The location of this tree in Fig. 3 with $\psi=180^{\circ}$ and $\psi=0^{\circ}$, respectively, moves to the left side of the damage pattern for low $v_{\text {crit }}$ and to the right side for high $v_{\text {crit }}\left(>70.0 \mathrm{~m} \mathrm{~s}^{-1}\right)$ with increasing absolute values of $\alpha$. Both cases lead to a convergent tree damage pattern. This convergent tree damage pattern is consistent with the inflow structure of the near-surface tornado wind field for $|\alpha|<90^{\circ}$ (cf. Fig. 2d).

At $|\alpha|=90^{\circ}$, trees close to $\psi=0^{\circ}$ occur on the right side of the damage pattern in Fig. 3. They indicate a divergence line and result from the dominating tangential flow of the tornado wind field. For $|\alpha|>90^{\circ}$, the locations of these trees move to the center of the damage pattern. The related wind field in Fig. $2 \mathrm{f}$ has a divergent structure demonstrating the outflow from the tornado vortex core. However, the separation into two calm points for $G_{\max } \geq 1$ in Figs. $2 \mathrm{c}-\mathrm{f}$ is not reflected in the tree damage patterns of Fig. 3 (cf. Letzmann 1923) because of the low wind speeds in these regions.
The tree damage patterns as a function of $G_{\max }$ and $\alpha$ have been classified by different swath types (Letzmann 1923, 1925). Figure 4 illustrates these tree damage patterns and corresponding streamline patterns for the different swath types. For $|\alpha|>90^{\circ}$, all swath types show a divergent damage pattern, while for $|\alpha|<90^{\circ}$, the convergent damage patterns of the individual swath types differ, depending on $G_{\max }$ and $v_{\text {crit. }}$. For values of $G_{\max }<1$, swath type I has a weak convergent damage pattern and is independent of the value of $v_{\text {crit }}$. For high values of $G_{\max }>3.5$, swath type IV is clearly identified by a tree with $\psi=180^{\circ}$ in the tree damage pattern independent of $v_{\text {crit }}$. Swath type II and swath type III occur for values of $G_{\max }$ between 1.0 and 3.5. Tree damage patterns classified as swath type II have small or moderate values of $v_{\text {crit }}\left(<70.0 \mathrm{~m} \mathrm{~s}^{-1}\right)$ and are indicated by locations of a tree with $\psi=0^{\circ}$ moving to the left side of the damage pattern for increasing $|\alpha|$. Swath type III is found for values of $v_{\text {crit }}>70.0 \mathrm{~m} \mathrm{~s}^{-1}$ indicated by locations of the tree with $\psi=0^{\circ}$ shifting to the right side of the damage pattern for increasing $|\alpha|$.

\section{b. Tree damage model}

Based on various tree parameters (for actual values see section 2c), like tree height $h_{t}$, tree diameter at breast height $d_{\mathrm{BH}}$, and modulus of rupture $M_{\text {rup }}$, the bending moment $B_{\max }$ and the tree resistance for stem breakage $M_{\text {crit }}$ are calculated and compared. From this comparison, $v_{\text {crit }}$ is evaluated. This algorithm is based on the HWIND model of Peltola and Kellomäki (1993). First, the forces contributing to $B_{\max }$ have to be considered. The tree is divided into $\Delta z=1-\mathrm{m}$ segments in the vertical. The areas of the stem and the crown are approximated by a rectangle and two isosceles triangles, respectively (Fig. 5). To calculate the drag force $F_{W}$ on the tree, a modified logarithmic wind profile (Prandtl 1925) within and above the forest was used to derive the horizontal velocity $v_{h}$ for each tree segment:

$$
\bar{v}_{h}=\frac{v *}{\kappa} \ln \frac{z}{z_{0}} \ln \frac{h_{t}}{z_{0}} .
$$

In Eq. (3), $v_{*}$ indicates the friction velocity, $z$ is the height above ground (and also the number of the tree segment), $z_{0}$ is the roughness length, and $\kappa=0.4$ is the

FIG. 8. Simulated damage patterns (lower left) are compared with the observed damage patterns (top) of the main vortex of the Milosovice tornado. From the simulated damage patterns, which are more regular than the observed ones, the tornado near-surface wind fields are derived. On the right, the corresponding F-scale area distribution along the path is shown. In all panels, tornado propagation is from bottom to top. 

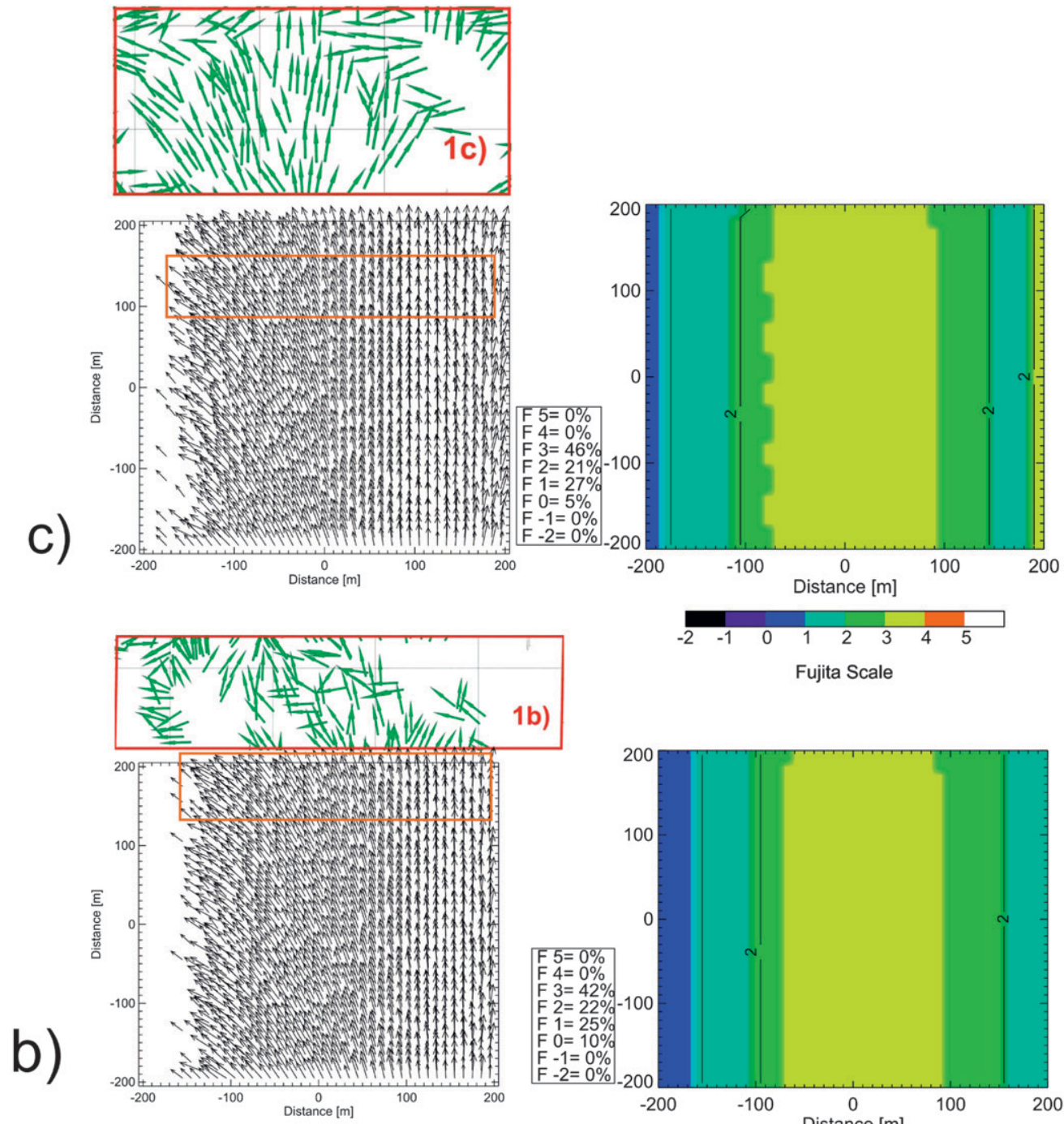

1b)

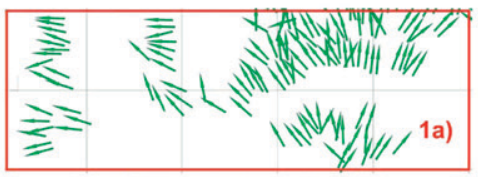
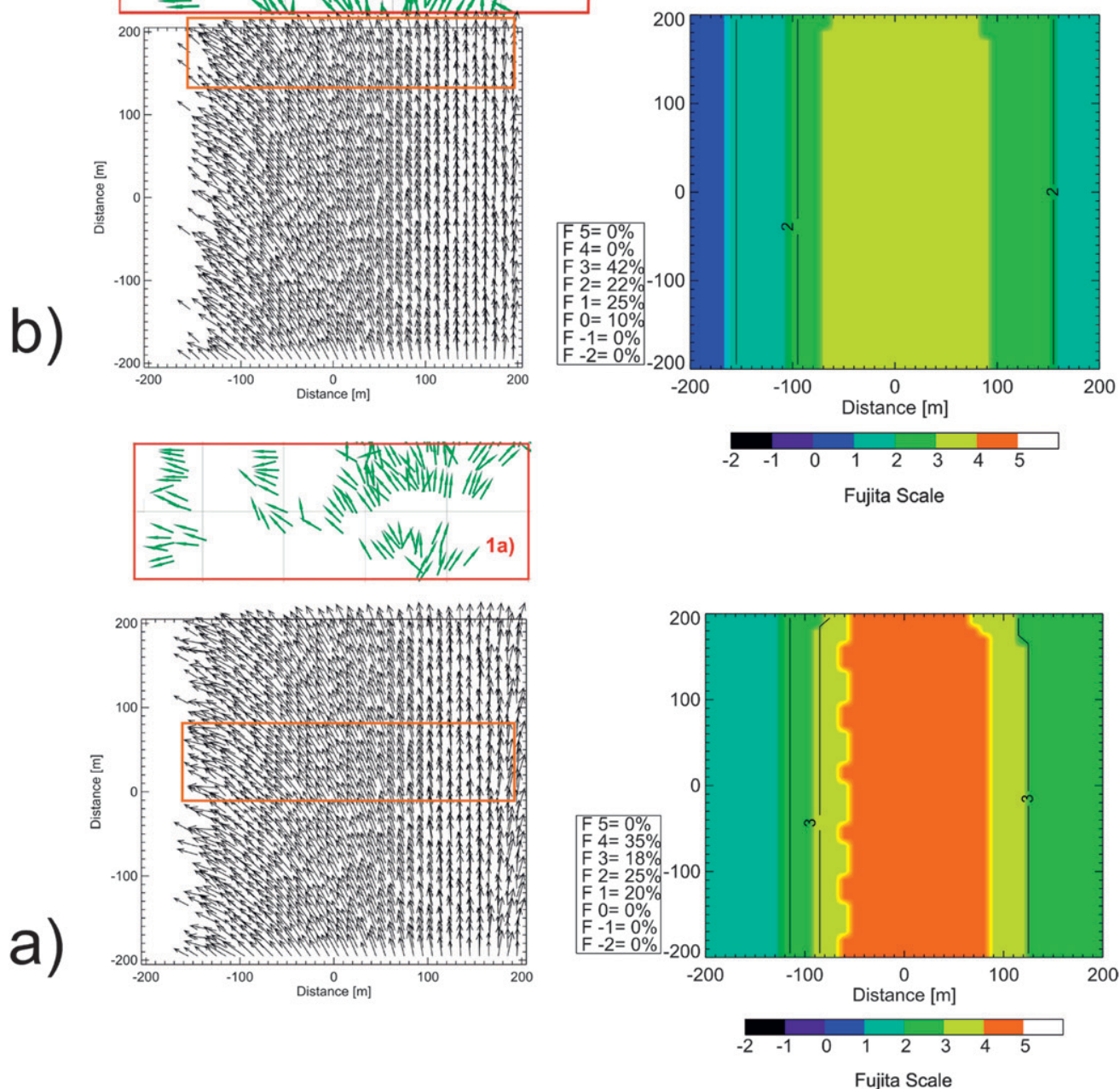
TABLE 2. Vortex parameters and velocity components derived from the damage analysis for the individual vortices of the Milosovice tornado. In most cases, a standard Rankine vortex was applied (cf. Beck 2008).

\begin{tabular}{ccccccc}
\hline \hline Vortex & $G_{\max }$ & $\alpha\left(^{\circ}\right)$ & $\begin{array}{c}R_{\max } \\
(\mathrm{m})\end{array}$ & $\begin{array}{c}v_{\text {trans }} \\
\left(\mathrm{m} \mathrm{s}^{-1}\right)\end{array}$ & $\begin{array}{c}v_{\text {cir }} \\
\left(\mathrm{m} \mathrm{s}^{-1}\right)\end{array}$ & $\begin{array}{c}v_{\max } \\
\left(\mathrm{m} \mathrm{s}^{-1}\right)\end{array}$ \\
\hline 1a & 5.0 & -140 & 80 & $16.5 \pm 1.0$ & $82.5 \pm 5.0$ & $99.0 \pm 6.0$ \\
1b & 4.0 & -140 & 80 & $16.5 \pm 1.0$ & $55.5 \pm 4.0$ & $82.5 \pm 5.0$ \\
1c & 4.0 & -150 & 80 & $16.5 \pm 1.0$ & $55.5 \pm 4.0$ & $82.5 \pm 5.0$ \\
2a & 1.5 & 160 & 80 & $9.8-17.8$ & $14.8-26.7$ & $24.7-44.5$ \\
2b & 1.5 & 180 & 80 & $9.8-17.8$ & $14.8-26.7$ & $24.7-44.5$ \\
2c & 1.0 & 180 & 80 & $12.3-22.3$ & $12.3-22.3$ & $24.7-44.5$ \\
3a & 1.0 & 0 & 40 & $12.4-22.3$ & $12.4-22.3$ & $24.7-44.5$ \\
3b & 2.5 & -90 & 40 & $7.1-12.7$ & $17.6-31.8$ & $24.7-44.5$ \\
3c & 4.0 & -90 & 60 & $4.9-8.9$ & $19.8-35.6$ & $24.7-44.5$ \\
4a & 4.0 & 150 & 60 & $4.9-8.9$ & $19.8-35.6$ & $24.7-44.5$ \\
4b & 4.0 & 120 & 60 & $4.9-8.9$ & $19.8-35.6$ & $24.7-44.5$ \\
\hline
\end{tabular}

von Kármán constant. The drag force for each 1-m segment is (Peltola and Kellomäki 1993)

$$
F_{W}(z)=\frac{1}{2} c_{d} \rho \bar{v}_{h}^{2} A(z),
$$

where $c_{d}$ indicates the drag coefficient, $\rho$ is the air density, and $A$ denotes the windward-projected cross section of each 1-m segment. If the velocity is high enough, the tree (i.e., foliage and smaller branches) will become streamlined and the effective cross-sectional area of the crown is reduced. For wind speeds less than $11.0 \mathrm{~m} \mathrm{~s}^{-1}$, $A$ is reduced by $20 \%$, while for wind speeds greater than $20.0 \mathrm{~m} \mathrm{~s}^{-1}$ the reduction increases to $60 \%$. In between, Peltola and Kellomäki (1993) used a streamlining factor $S_{t}$ to describe the reduction:

$$
S_{t}(z)=\frac{10.0}{\bar{v}_{h}(z)}-0.10
$$

If the tree not only becomes streamlined but also starts to bend over for higher wind speeds, the gravitational acceleration $g$ leads to a force $F_{G}$ on the overhanging crown:

$$
F_{G}(z)=m_{c} g
$$

The crown green mass $m_{c}$ and the stem mass follow from Baldwin (1987). The tree deflection $x$ and $B_{\max }$ per segment are again based on Peltola and Kellomäki (1993):

$$
B_{\text {max }}(z)=f_{\text {gust }} f_{\text {gap }}\left[F_{W}(z) \Delta z+F_{G}(z) x(z)\right]
$$

The gust factor $f_{\text {gust }}$ indicates the ratio between the maximum and the mean bending moment and was determined from wind tunnel experiments (Gardiner et al. 1997), while the gap factor $f_{\text {gap }}$ characterizes the effects of variable upwind gaps in the tree stand (Holland et al. 2006). The total bending moment for each tree is the sum of all bending moments per tree segment and is compared to $M_{\text {crit }}$ (Peltola and Kellomäki 1993) to determine $v_{\text {crit }}$ :

$$
M_{\text {crit }}=\frac{\pi}{32} M_{\text {rup }} d_{\mathrm{BH}}^{3} .
$$

\section{c. Model implementation}

The model for the simulation of tree damage patterns consists of a wind field module and a tree damage module. The structure of the model and the module interrelations are outlined in Fig. 6. In the typical setup, the calculations are done for a $400 \mathrm{~m} \times 400 \mathrm{~m}$ horizontal domain with a grid spacing of $\Delta x=\Delta y=10 \mathrm{~m}$. For the tree module, the domain is extended to three dimensions with a height of $30 \mathrm{~m}$ and a grid size of $\Delta z=1 \mathrm{~m}$ in the vertical direction.

In the first part of the model, $v_{\text {crit }}$ is derived. The bending moment and the tree resistance are calculated from an iterative process, using several tree parameters and an initial guess for the wind speed. If the bending moment exceeds the tree resistance, the iteration ends, otherwise the velocity is incremented by $0.5 \mathrm{~m} \mathrm{~s}^{-1}$ steps. According to the HWIND model (Peltola and Kellomäki 1993), the tree resistance for stem breakage can be replaced by the tree resistance for overturn to calculate the critical velocity for tree overturn $v_{\text {crit }, o}$. If tornado damage patterns with only uprooted trees occur, the appropriate tree resistance for tree overturn is used for the simulation of tree damage patterns (Beck 2008).

Values of $v_{\text {crit }}$ calculated individually for each tree are then used by the wind field module to simulate the tree damage patterns. The wind field module produces an instantaneous velocity at each grid point that is compared with $v_{\text {crit }}$. If the instantaneous velocity of the wind field exceeds $v_{\text {crit }}$, the tree is considered to be downed, and the fall direction is assumed to be the instantaneous direction of the wind field at the corresponding point, in accordance with Letzmann (1923), Holland et al. (2006), and Bech et al. (2009).

The vortex representing the tornado wind field starts at the southern domain boundary and then moves across the domain in positive $y$ direction with translation speed $v_{\text {trans }}$. To avoid undersampling, the time step $\Delta t$ was limited to

$$
\Delta t \leq \frac{\Delta y}{v_{\text {trans }}} .
$$




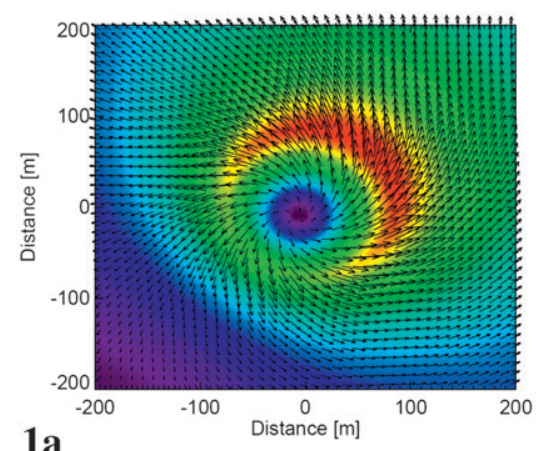

$1 \mathrm{a}$
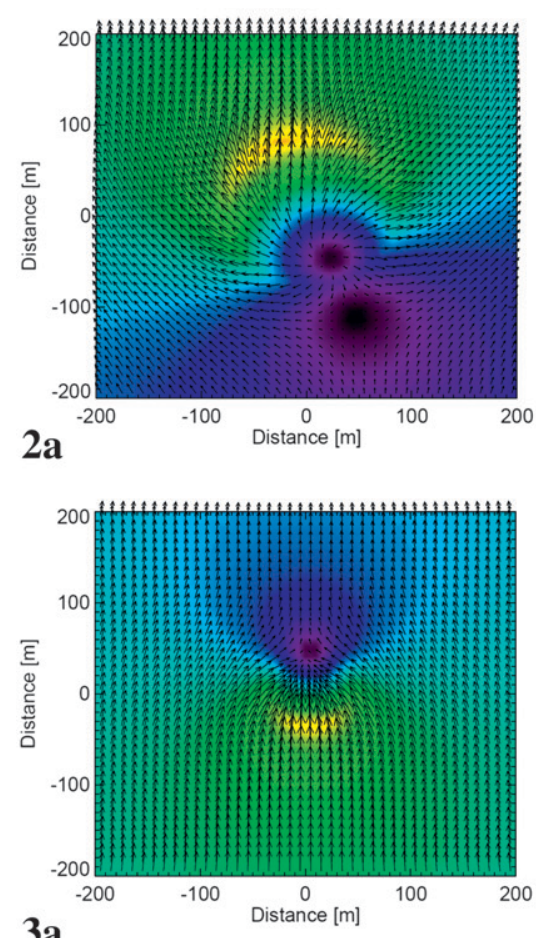

3a

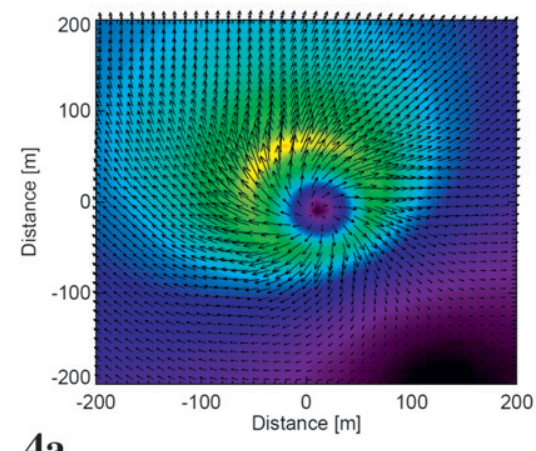

$4 a$

FIG. 9. Reconstructed near-surface tornado wind fields $\left(\mathrm{m} \mathrm{s}^{-1}\right)$ from the Milosovice damage patterns for (top) the main vortex 1 and (second from top)-(bottom) the three smaller vortices 2-4 indicating spiral outflow for the vortices 1,2 , and 4 . Vortices 1 and 3 have cyclonic senses of rotation, while vortices 2 and 4 were anticyclonic. For the simulation, a standard Rankine vortex was used. 


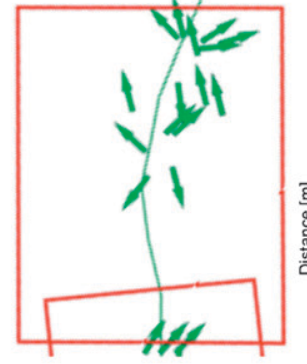

V)

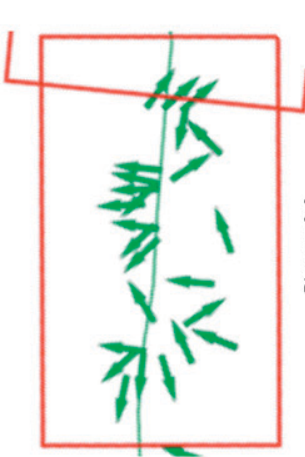

IV)

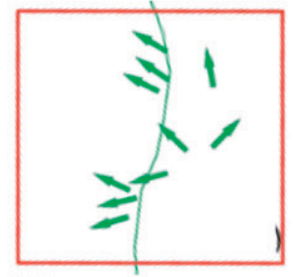

III)

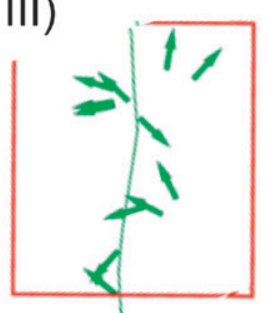

II)

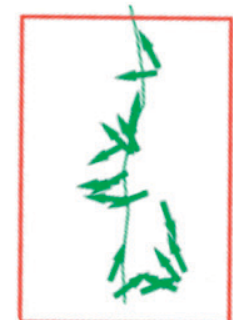

I)
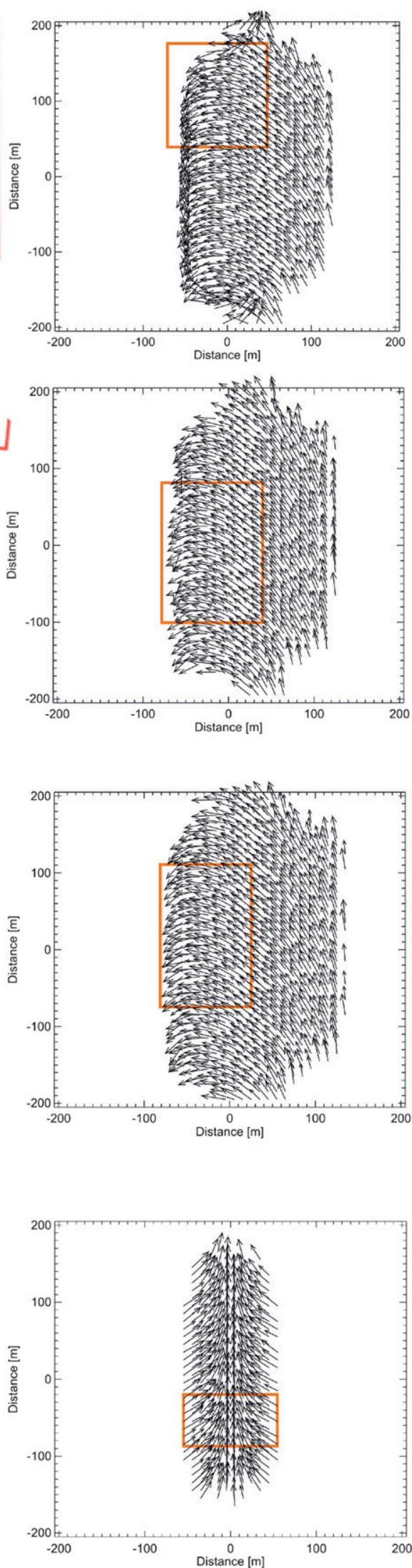
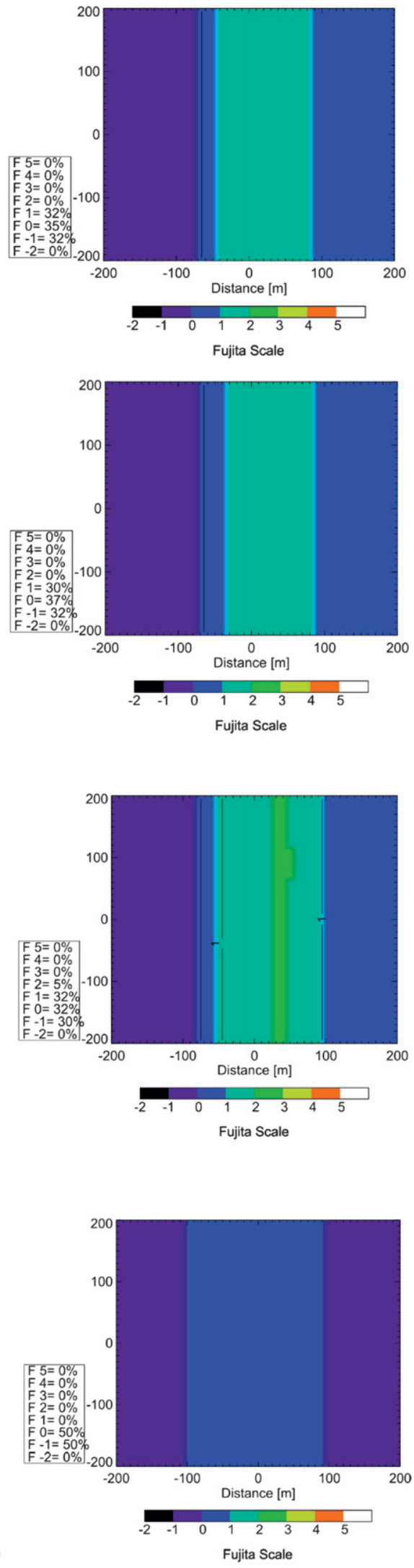
TABLE 3. Vortex parameters and velocity components derived from the damage analysis of the Castellcir tornado. For all simulations, a standard Rankine vortex was applied (cf. Beck 2008).

\begin{tabular}{lcrcccc}
\hline \hline Region & $G_{\max }$ & $\alpha\left(^{\circ}\right)$ & $\begin{array}{c}R_{\max } \\
(\mathrm{m})\end{array}$ & $\begin{array}{c}v_{\text {trans }} \\
\left(\mathrm{m} \mathrm{s}^{-1}\right)\end{array}$ & $\begin{array}{c}v_{\text {cir }} \\
\left(\mathrm{m} \mathrm{s}^{-1}\right)\end{array}$ & $\begin{array}{c}v_{\max } \\
\left(\mathrm{m} \mathrm{s}^{-1}\right)\end{array}$ \\
\hline I & 2.0 & 0 & 50 & $11.0 \pm 1.0$ & $22.0 \pm 2.0$ & $33.0 \pm 3.0$ \\
II & 4.0 & -90 & 50 & $11.0 \pm 1.0$ & $44.0 \pm 4.0$ & $55.0 \pm 5.0$ \\
III & 4.0 & -90 & 50 & $11.0 \pm 1.0$ & $44.0 \pm 4.0$ & $55.0 \pm 5.0$ \\
IV & 3.5 & -90 & 50 & $11.0 \pm 1.0$ & $38.5 \pm 3.5$ & $49.5 \pm 4.5$ \\
V & 3.5 & -70 & 50 & $11.0 \pm 1.0$ & $38.5 \pm 3.5$ & $49.5 \pm 4.5$ \\
\hline
\end{tabular}

Initial conditions for the tree and the wind field module have to be specified as well. For the tree module, either a random or a homogeneous distribution of tree age $a$ has been provided. Furthermore, various tree parameters depending on the tree species have to be set, for instance for Norway spruce (picea abies): $h_{t}=24.6 \mathrm{~m}$, $a=70 \mathrm{yr}, d_{\mathrm{BH}}=0.30 \mathrm{~m}, M_{\mathrm{rup}}=39.1 \mathrm{MPa}$, modulus of elasticity $E=7000 \mathrm{MPa}$, gap size $g_{s}=15 h_{t}$ (accounting for the upwind gap or fetch on the forest edge in the calculation of $f_{\text {gap }}$ ), and $c_{d}=0.29$. For the calculation of $f_{\text {gust }}$, the distance between two trees is set to $2.5 \mathrm{~m}$, so every fourth tree is resolved by the model grid.

For the wind field module, both $G_{\max }$ and $\alpha$ depend on the simulated velocity field. The tornado core is set to $R_{\max }=75 \mathrm{~m}$, and $v_{\max }=80.0 \mathrm{~m} \mathrm{~s}^{-1}$ (including $v_{\text {trans }}$ ). Finally, the coordinates of the starting point of the vortex center are set to $\left(x_{c}, y_{c}\right)=(0,-200) \mathrm{m}$.

\section{d. Model validation}

The theoretical tree damage patterns of Letzmann (1923) are compared with the tree damage patterns produced by the model in Fig. 3 for $G_{\max }=2.0$ and different $\alpha$. Letzmann (1923) derived tree damage patterns for each $\alpha$ for four different tree resistances, varying $v_{\text {crit }}$ as multiples of the translation velocity of the tornado (Fig. 3a). Our reproduced tree damage patterns in Fig. 3b (cf. Beck 2008) have been simulated for three different magnitudes of $v_{\text {crit }}$ calculated from the tree module. Figure $3 \mathrm{~b}$ shows the location of a tree with $\psi=180^{\circ}$ for $|\alpha|<90^{\circ}$ moving to the left side of the tree damage pattern for small $v_{\text {crit }}$ in the same way as depicted in Fig. 3a. For high $v_{\text {crit }}$, in Figs. 3a and 3b, the width of the damage path is reduced and the tree location with $\psi=0^{\circ}$ moves to the left for $|\alpha|<90^{\circ}$. Crossed trees at $|\alpha|=60^{\circ}$ occur in Figs. 3a and $3 \mathrm{~b}$ as well. For $|\alpha|>90^{\circ}$, a divergent damage pattern with a tree location having $\psi=0^{\circ}$ that moves to the left flank of the track is also notable for both the Letzmann (1923) graph and our simulation.

The absolute values of $v_{\text {crit }}$ for both tree damage patterns do not correspond exactly, as our absolute value of $v_{\text {crit }}$ for moderate and high intensities in Fig. $3 \mathrm{~b}$ is presumably smaller compared to Letzmann's values in Fig. 3a. Yet, in summary, we argue that the structure of the damage patterns from Letzmann (1923) and Beck (2008) are very similar in cases of equal values for $v_{\text {crit }}$. Now the developed model can be applied to determine tornado intensities from forest damage and comparison with other models, like those of Holland et al. (2006) or Bech et al. (2009).

\section{Forest damage analyses}

To reconstruct tornado near-surface wind fields and to determine tornado intensities from forest damage patterns, the observed tree damage patterns of two tornadoes are analyzed here with respect to their relevant parameters. Further applications of our model were presented by Beck (2008). Note that to use this method for the wind field reconstruction of tornadoes, it is essential to either rely on aerial photographs (cf. Dotzek et al. 2007; Dotzek and Friedrich, 2009) of the forest damage patterns or Letzmann's "method of lines" in ground surveys, as already emphasized by Letzmann (1939). Otherwise, the inherent parallax errors will make it rather difficult to determine the location of the fallen trees with the necessary precision. If this condition is satisfied (as in our two cases below), then the general procedure for applying our model to an actual forest damage observation is as follows.

First, an estimation of $\alpha$ is done by inspecting if the damage patterns are convergent $\left(|\alpha|<90^{\circ}\right)$ or divergent $\left(|\alpha|>90^{\circ}\right)$. From this estimation, the stage of the tornado life cycle (Letzmann 1923; Davies-Jones 1986) can readily be identified. The sense of vortex rotation (cyclonic or anticyclonic) is derived from comparison of the damage patterns to the cyclonic swath types of Letzmann (1923; cf. Fig. 4 herein). From radar or ground observations, the translation direction is inferred and $v_{\text {trans }}$ is

FIG. 10. Analysis of the Castellcir tornado. (left) The location of the broken trees (after Bech et al. 2009) chronologically divided into regions I-V. (middle) The simulated tree damage patterns, as well as the boxes used for comparison with the observed damage pattern. (right) The F-scale area distribution for each region. Because the obtained parameters from regions II and III are identical, only one simulation was performed. In all panels, tornado propagation is from bottom to top. 
calculated, using storm propagation as a proxy for tornado translation. In the next step, downed trees with $\psi=0^{\circ}$ and $\psi=180^{\circ}$ with respect to the direction of translation have to be identified in the observed forest damage patterns. From the number of convergence or divergence lines reflected in the pattern of broken trees, the values $G_{\max }$ and $\alpha$ can be quantified. We estimate $R_{\max }$ from the width of the damage path in consecutive trial simulations. The observed damage patterns using the tree parameters from section $2 \mathrm{c}$ have to be simulated iteratively by varying the initial estimates of $G_{\max }, \alpha$, and $R_{\max }$. From this, the structure and intensity of the tornado wind field is recalculated until the qualitative agreement between the simulated tree fall pattern and the observed damage swath is maximized.

\section{a. Milosovice tornado, 31 May 2001}

An F3 tornado occurred near Milosovice-Velka Paseka in the Czech Republic on 31 May 2001 with a path width of 400-500 m and pathlength of $16 \mathrm{~km}$ (www.essl. org/ESWD/; cf. Dotzek et al. 2009). Besides the main vortex, three smaller vortices were observed (www. chmi.cz/torn/cases/20010531/20010531.html; M. Setvák 2008, personal communication). The translation velocity of the thunderstorm cell producing the tornado was estimated from Czech Hydrometeorological Institute (CHMI) radar observations (not shown) as $16.5 \pm$ $1.0 \mathrm{~m} \mathrm{~s}^{-1}$, which is also assumed as $v_{\text {trans }}$ of the tornado. ${ }^{1}$ The radar observations confirmed that the thunderstorm propagated to the east-southeast, in line with the main tornado damage swath. The aerial photo of the forest damage (Fig. 7a) shows the division of the forest damage patterns according to the four vortices. The main vortex (1) is identified with the tornado, while the damage patterns of the other three vortices (2-4) surround the damage pattern of the main vortex.

The comparison between the simulated and the observed damage patterns of the main tornado is illustrated in Fig. 8 as well as the distribution of the F scale along the damage path for these simulations. By comparing the observed damage patterns of the main tornado with the swath types of Fig. 4, a divergent damage pattern has been found. This evidence supports $90^{\circ}<$ $|\alpha|<180^{\circ}$. By variation of $G_{\max }$ and $R_{\max }$, the observed damage pattern is approached in consecutive simulations. For the simulations, the tree parameters for Norway spruce as mentioned in section $2 \mathrm{c}$ have been used. Comparing the three damage patterns of the main

\footnotetext{
${ }^{1}$ In the error calculation in section 4 , we also consider the effects of a discrepancy between the tornado propagation and the translation of the thunderstorm cell.
}

vortex, Fig. 8a shows a more divergent damage pattern than Figs. $8 \mathrm{~b}$ and $8 \mathrm{c}$. The width of the damage path is constant throughout the damage pattern, leading to a constant $R_{\max }$ of the vortex core. The best correlation to the observed damage pattern can be found for $G_{\max }=$ $4.0-5.0,|\alpha|=140^{\circ}-150^{\circ}$, and $R_{\max }=80 \mathrm{~m}$. Reasons for the not completely exact match of the observed and simulated damage patterns might be that the model does not take into account any interaction of falling trees. Further, terrain effects that might lead to a higher damage level downhill or a lower damage level uphill due to frictional and gravitational forces are also not included in the model. Therefore, not all variation in the structure of the damage patterns can be reproduced by the model. In the Milosovice tornado, parts of the broken trees were also observed on a downsloping terrain, which probably caused stronger damage and slightly different treefall patterns compared to our simulation.

For vortex 2, an anticyclonic rotation and a strongly divergent damage pattern are notable. Vortex 3 is found to have smaller spatial extension and a cyclonic sense of rotation similar to the main vortex. Further, a central convergence line and a fast-changing structure of the damage patterns have been detected. Vortex 4 shows anticyclonic rotation with a strongly divergent damage pattern. A detailed overview of the parameters used for the simulations of the damage patterns of the different vortices is given in Table 2 .

From the simulation of the damage patterns, the vortex trace (centerline of damage swath) and its divergence and convergence lines can be located. Thus, we now focus on the location of the trace as well as these divergence and convergence lines as illustrated in Fig. $7 \mathrm{~b}$ for all four vortices. In vortices 1,2 , and 4 , the divergence line is indicated by a tree with $\psi=0^{\circ}$, while for vortex 3 , the tree with $\psi=0^{\circ}$ indicates a convergence line. Considering vortices 1 and 4 , the divergence line is located on the right and left side of the trace of the tornado, respectively. For vortices 2 and 3, the divergence and convergence lines, respectively, coincide with the trace of the vortex.

From the simulation, the maximum velocity of the main vortex is derived by using $v_{\text {trans }}$ of the main vortex determined by radar observations and the relation among $v_{\text {cir }}, v_{\text {trans }}$, and $G_{\max }($ Letzmann 1923):

$$
G_{\max }=v_{\text {cir }} / v_{\text {trans }} .
$$

For the three smaller vortices around the main vortex, the individual translation velocity components are unknown. Nevertheless, a lower limit of the maximum velocity can be calculated based on $v_{\text {crit }}$ from the tree module for these vortices. A summary of all calculated velocity components is given in Table 2 . 
Figure 9 illustrates the reconstructed near-surface wind fields corresponding to the four vortices. The wind fields of the main vortex 1 (top) illustrate the largest spatial extension of all vortices. Its structure does not change significantly. An anticyclonic rotation in the wind fields of vortex 2 (second from top) is shown. The reconstructed wind fields of vortex 3 (second from bottom) show parts of the evolution of a tornado life cycle: the pattern changes from a convergent inflow in the organizing and formation stage of a tornado to a pure tangential flow in the mature stage of a tornado (cf. Hall and Brewer 1959). The wind fields of vortex 4 (bottom) are similar to those of the main vortex, but with smaller spatial extension and anticyclonic rotation.

From the reconstructed near-surface wind fields, an F-scale distribution within the domain can be derived. Figure 8 shows the resulting distribution for the main vortex indicating a widespread F3 zone (71-93 $\mathrm{m} \mathrm{s}^{-1}$ ) and even an F4 zone (93-117 $\mathrm{m} \mathrm{s}^{-1}$ ) for one of the damage patterns of the main vortex with the most widespread and extensive damage. Two of three damage patterns yield a peak intensity of $\mathrm{F} 3$, and damage of $\mathrm{F} 4$ intensity is limited to only a few points. The classification of the main vortex as a tornado is verified by obtaining a characteristic divergent damage pattern at the end stage of a tornado life cycle and a path width consistent with high-F3 tornadoes (Brooks 2004). Thus, in total, our model verifies the classification of the tornado as an F3 tornado.

\section{b. Castellcir tornado, 18 October 2006}

The F2 Castellcir tornado (Aran et al. 2009; Bech et al. 2009) occurred in Catalonia, Spain, on 18 October 2006 with a damage pathlength of approximately $4 \mathrm{~km}$ from southwest to northeast and a maximum damage path width of $260 \mathrm{~m}$. After the tornado, a microburst occurred farther to the northeast. In this case, a damage analysis was performed along the total damage path, allowing for a more detailed determination of tornado intensity compared to the analysis of only a single prominent part of the damage path, like for the Milosovice tornado. The $v_{\text {trans }}$ of the Castellcir thunderstorm cell was derived from radar observations as $11.1 \mathrm{~m} \mathrm{~s}^{-1}$ [see the detailed analysis of the tornado and its damage by Bech et al. (2009)]. In particular, it is fortunate that Bech et al. (2009) used a Letzmann-type model similar to ours, so comparing our analysis of the Castellcir tornado with their results will serve as additional verification of our model and help to substantiate the intensity assessment for this case.

Figure 10 (left column) illustrates the reported locations of the downed trees along the tornado damage path in five selected regions. This can be compared with Bech et al. (2009, their Fig. 8) showing the full damage swath and grouping it slightly differently in eight subregions. Their region 1 (F0 intensity) corresponds to our region I, while their regions 3 and 4 (F1 and F2 intensity) relate to our regions II and III, and finally, their regions 6 and 7 (F1 intensity) roughly correspond to our regions IV and V. Bech et al. (2009) did not derive $G_{\max }$ and $\alpha$ in all regions of the damage path, but they reported $G_{\max }=$ 2.0, $\alpha=0^{\circ}$ in region 1 , and $G_{\max }=4.0, \alpha=-90^{\circ}$ in region 4.

Our modeling results for the tree damage patterns (Fig. 10, middle column) corroborate and extend these results. A summary of all derived velocity components is shown in Table 3 while the tree parameters used for the simulations are the same as in section $2 \mathrm{c}$. The results show for region I a convergent damage pattern $\left(\alpha=0^{\circ}\right)$ typical for the beginning of a tornado life cycle, while regions II-V are dominated by mainly tangential flow $\left(|\alpha| \simeq 90^{\circ}\right)$. Also, our derived values for $G_{\max }$ are well in line with Bech et al. (2009). Yet, we were able to obtain the vortex parameters in a larger number of path regions. From the derived parameters, the F-scale distribution along the path (Fig. 10, right column) provides evidence for first F0 and then mostly F1 intensity with a very small embedded F2 zone in regions II and III, which is exactly in line with Bech et al. (2009). Therefore, the $\mathrm{F} 2$ rating of the tornado is verified even if the F2 zone is very small (Beck 2008).

In addition, the cyclonic sense of rotation of the tornado was also confirmed, and $R_{\max }=50 \mathrm{~m}$ in this case is smaller compared to $R_{\max }$ of the Milosovice tornado. Note that terrain effects not included in the model might have played an important role for the specific local structure of the damage patterns, as the terrain elevation along the tornado track varied by approximately $200 \mathrm{~m}$ (Bech et al. 2009).

\section{Discussion}

The model developed here allows for a more accurate determination of the tornado intensity in comparison with the classification of tornado intensity based on pure damage analysis (Fujita 1981). Additionally, detailed information about the type of the tornado near-surface wind field and the location of the tornado trace are gained. From the simulation and comparison of the tree damage patterns to the observed damage patterns, $G_{\max }, \alpha$, and $R_{\max }$ are derived. Together with the translation velocity of the tornado as evaluated from radar or ground observations, the maximum intensity of the tornado can be calculated. In our cases, the thunderstorm translation speed was determinable with an uncertainty of $\pm 1.0 \mathrm{~m} \mathrm{~s}^{-1}$. This also takes into account small deviations of the tornado propagation from the translation of its 

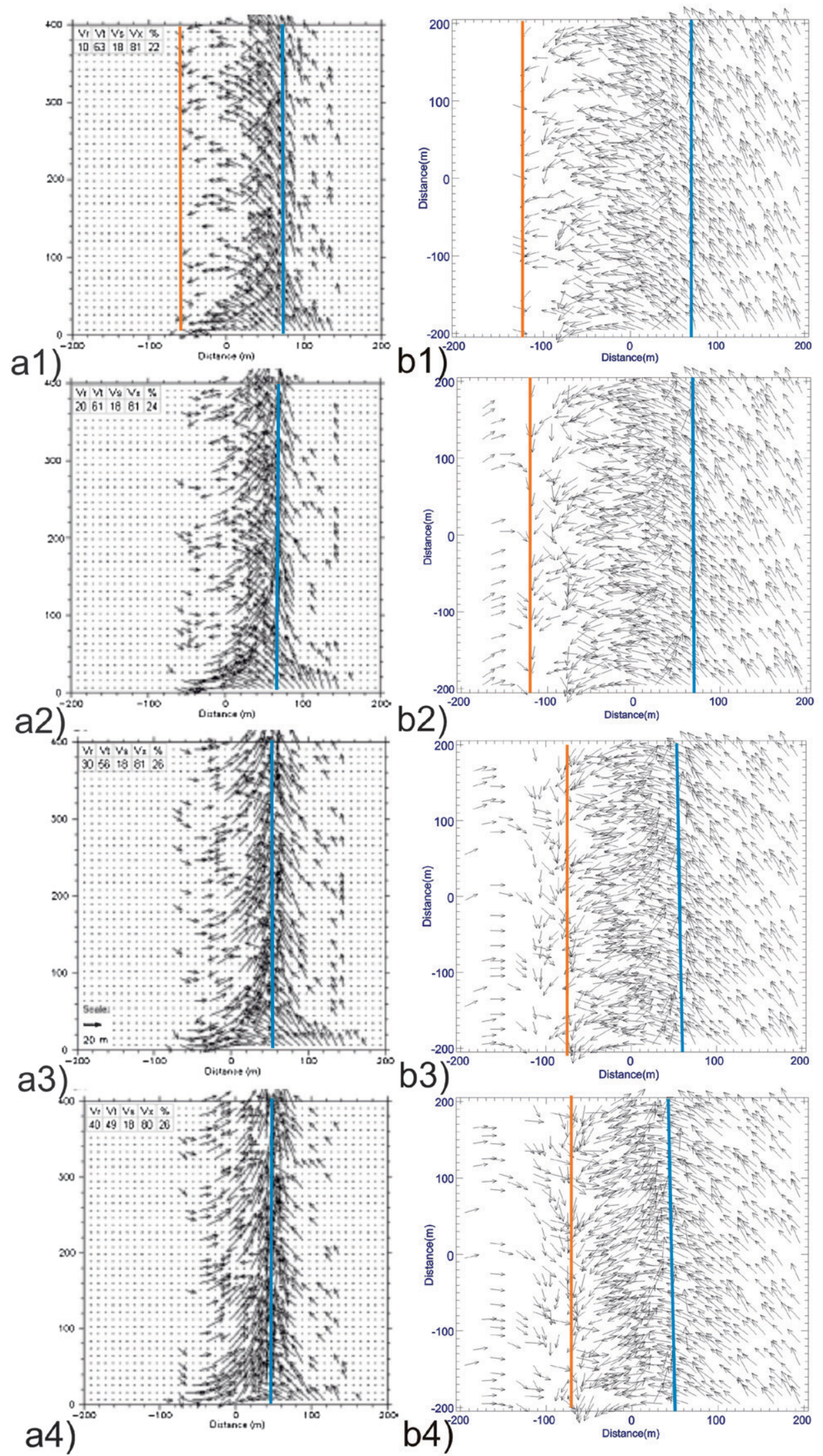

b2)

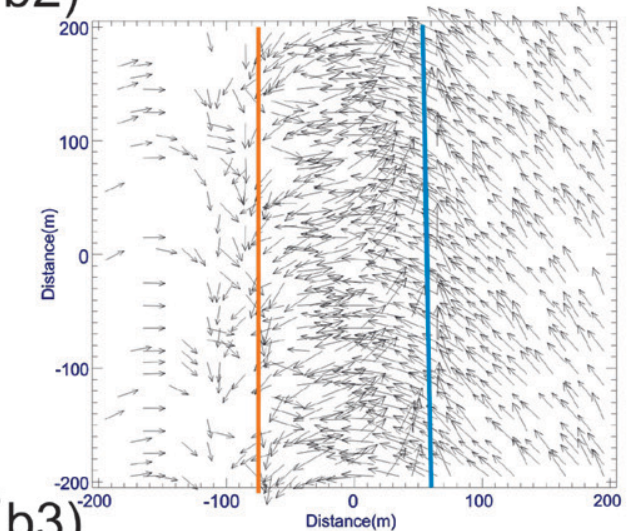
b3)

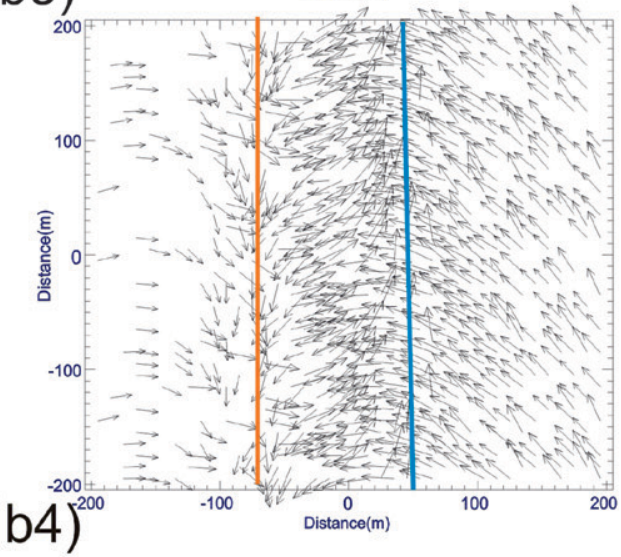


parent thunderstorm cell, for instance, by weak meandering of the tornado. The error in the estimation of the maximum tornado velocity can readily be tied to the uncertainty of the translation velocity (Beck 2008):

$$
\Delta v_{\max }=\left(G_{\max }+1\right) \Delta v_{\text {trans }} .
$$

As $G_{\max }$ does not appear to exceed values of 6.0 [already suggested by Letzmann $(1923,1925)$, and consistent with our cases peaking at $\left.G_{\max }=5.0\right]$, the highest intensity of the Milosovice tornado is determinable with a maximum error of $\Delta v_{\max } \approx \pm 6.0 \mathrm{~m} \mathrm{~s}^{-1}$. This accuracy is less than one half-step of the F scale (Table 1). With prior knowledge of the translation velocity and the subsequent derivation of the relevant tornado parameters using our model, the near-surface wind field of the tornado can be completely reconstructed, and conclusions on the life cycle stage of the tornado from confluent early stages to the more diffluent decay can be drawn.

Provided the translation speed is known, the biggest advantage of the Letzmann-type model for tornado cases is its independence from the tree species and other tree parameters (Beck and Dotzek 2009). For tornado vortices, the structure of the damage patterns itself already allows the reconstruction of all relevant wind field parameters, provided that a sufficient amount of trees was downed. So in this case, the detailed tree model is unnecessary, and the inherently high uncertainties of tree models with respect to wood parameters, tree species, age distribution, or even soil type and moisture can be excluded. The only necessary parameter for simulating tree damage patterns is an average value for $v_{\text {crit }}$. In our case, it was derived from the tree model. An evaluation of the uncertainties in $v_{\text {crit }}$ from the tree module based on the HWIND model was given by Beck (2008): to adapt the HWIND model to more realistic conditions, a Gaussian distribution of $M_{\text {rup }}$ was introduced. This led to an uncertainty of $\Delta v_{\text {crit }}=20.0-31.0 \mathrm{~m} \mathrm{~s}^{-1}$ for a $90 \%$ confidence level of $M_{\text {rup }}$, as deemed necessary to describe the conditions in tree stands realistically.

Of course, such uncertainties larger than one full step of the $\mathrm{F}$ scale lead to a less reliable determination of tornado intensities. Instead, the independence of tree parameters in tornado cases gives our model its accuracy. Note also that our model is not limited to the simulation of forest damage patterns for tornado intensity determination. It can likewise be used to simulate crop damage patterns to determine the tornado intensity, as already argued by Letzmann (1923).

When compared with the simulated tornadic forest damage patterns of Holland et al. (2006), our model shows some notable differences. In both cases, the tree module is based on the HWIND model of Peltola and Kellomäki (1993). However, contrary to the random age distribution of trees used by Holland et al. (2006), both random and homogeneous age distributions of either loblolly pine (pinus taeda) or Norway spruce (picea abies) are used, as homogeneous age distributions better fit the conditions of many forests in Europe (Letzmann 1923; Hubrig 2004; M. Hubrig 2008, personal communication).

Holland et al. (2006) referred to Letzmann (1923) but used $v_{r}, v_{\theta}$, and $v_{\max }$ to describe a Rankine vortex for their simulation of forest damage patterns. In our model, we used the full analytical tornado model and resulting theoretical tree damage patterns by Letzmann (1923), which rely on $G_{\max }, \alpha$, and $v_{\max }$ to describe the Rankinetype vortex. Figure 11 shows that the structure of the damage patterns of Holland et al. (2006) and our simulated tree damage patterns is similar. Yet, because of the dependence of their tornado wind field on the parameters $v_{r}, v_{\theta}$, and $v_{\max }$, only values of $51^{\circ} \leq|\alpha| \leq 90^{\circ}$ have been considered by Holland et al. (2006). With our present model, the structure of divergent tornado damage patterns occurring for $90^{\circ}<|\alpha|<180^{\circ}$ can also be simulated.

In comparing Fig. 11 with the theoretical damage patterns of Fig. 3a, we found that the simulated damage patterns of Holland et al. (2006) correspond to a high value of $v_{\text {crit }}$ while our simulated damage patterns correspond to a moderate value of $v_{\text {crit. }}$. This is confirmed by the number of convergence lines in the simulated damage patterns. The damage patterns of Holland et al. (2006) show only one convergence line with a tree of $\psi=$ $0^{\circ}$ while our damage patterns show two convergence

FIG. 11. Comparison of the simulated damage patterns of (a) Holland et al. (2006) and (b) the present study for a fixed $G_{\max }=3.5$ and (a1),(b1) $\alpha=80^{\circ}$, (a2), (b2) $\alpha=73^{\circ}$, (a3),(b3) $\alpha=63^{\circ}$, and (a4),(b4) $\alpha=$ $51^{\circ}$. From (a1),(b1) to (a4),(b4) $v_{r}$ increases, while $v_{\theta}$ decreases and $v_{\text {trans }}$ is held constant. There are more broken trees in (b) relative to (a), although the structure of the tree damage patterns is very similar. The blue lines indicate the convergence lines identified by a tree with $\psi=0^{\circ}$, while the orange convergence line is identified by a tree with $\psi=180^{\circ}$. The characteristic convergent tree damage patterns for inflow into the vortex center for $|\alpha|<90^{\circ}$ are notable in (a) and (b). A random distribution of tree age is used in (a) and (b). In all panels, the tornado propagation is from bottom to top. 

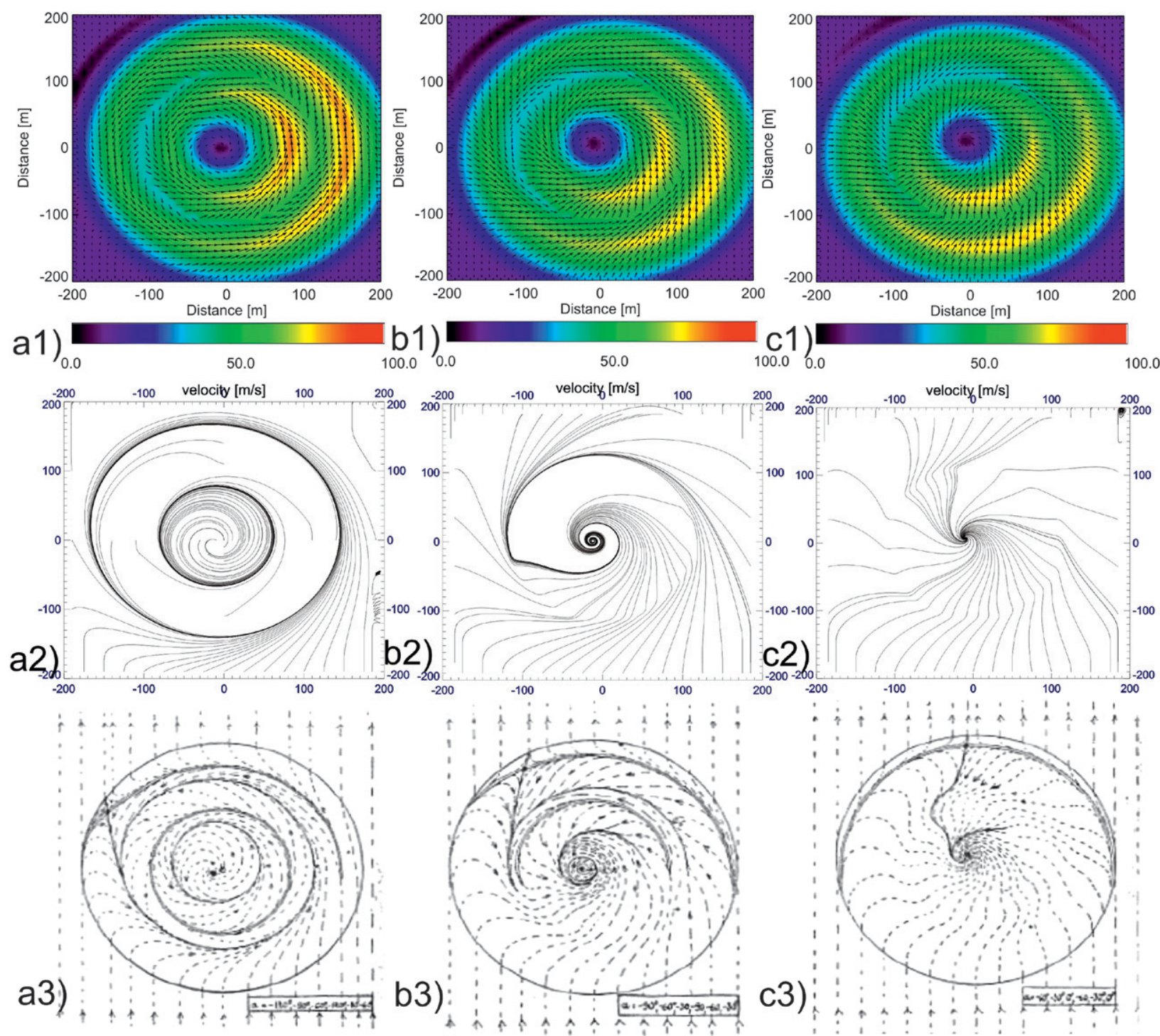

FIG. 12. (a1)-(c1) Wind fields ( $\mathrm{m} \mathrm{s}^{-1}$ ) and (a2)-(c2) streamline patterns produced by the present wind field module compared with (a3)(c3) streamline patterns from Letzmann (1923) for a tornado with double wind speed maximum. Accordingly, $\alpha$ increases and decreases twice. The starting angle $\alpha$ in the vortex center increases from (a1)-(a3) $-120^{\circ}$ to (b1)-(b3) $-90^{\circ}$ and (c1) $-(\mathrm{c} 3)-60^{\circ}$.

lines, one with a tree of $\psi=0^{\circ}$ and another with a tree of $\psi=180^{\circ}$. The damage patterns of Holland et al. (2006) contain a smaller number of broken trees. This might be due to the fact that either their value of $v_{\text {crit }}$ for the random-age forest was higher, or that their simulation time steps exceeded Eq. (9), leading to undersampling and a noncontinuous interaction between the near-surface tornado wind field and the tree stand.

Bech et al. (2009) have already used a Letzmann-type Rankine vortex depending on $G_{\max }, \alpha$, and $v_{\max }$ for their simulation of the tornado wind fields in the Castellcir tornado. In contrast to our procedure, they determined their values of $G_{\max }$ and $\alpha$ from comparison of the observed tree damage patterns with the simulations of the tornado wind field. With our simulations of the Castellcir tree damage patterns, we can verify the values for $G_{\max }$ and $\alpha$ as evaluated by Bech et al. (2009) and also obtain values of $G_{\max }$ and $\alpha$ for zones not classified by these authors: $G_{\max }=3.5$ and $\alpha=-90^{\circ}$ (zone IV) and $\alpha=$ $-70^{\circ}$ (zone V), respectively.

An additional comparison by Beck (2008) of the tree damage patterns resulting from an idealized downburst simulation to those of tornadoes revealed that it is possible to obtain damage patterns of almost identical appearance. But some distinguishing features between tornado and downburst damage in forests could be identified 
using our model. The damage patterns of the downburst consistently show a divergent structure, while the damage pattern of the tornado usually changes from a convergent structure in the formation stage to a more divergent structure in the following stages (Letzmann 1923). The fall angle $|\psi|$ of a divergent tornado damage pattern does not exceed $45^{\circ}$, whereas it does for the downburst, because here the wind field is more divergent and the translation velocity is smaller. A third but weaker characteristic is the spatial extension of the swath, that is, its length-to-width aspect ratio, which is generally larger for a tornado than for a downburst (e.g., Knupp 2000). The Milosovice tornado could be verified as a tornado event, because of small fall angles in the divergent structure of the damage pattern and a path aspect ratio typical of tornadoes (cf. Brooks 2004). Thus, simulation of the tree damage patterns also helps to discriminate between tornado and downburst events.

There are further applications of the concepts presented here, for instance, Letzmann's analytical model can also be applied to analyze the wind field structure of tropical and extratropical cyclones (Letzmann 1925). In addition, Letzmann $(1923,1925)$ had already analytically examined tornado vortices with two velocity maxima in $v_{r}$ and $v_{\theta}$. In Fig. 12, the velocity field and the streamline patterns of a wind field with double velocity maxima are illustrated. Note that the streamlines in Fig. 12b(2) show a surprising similarity to the shape of a certain class of hook echoes in radar observations of severe thunderstorms. Hook echoes are often found at low- or midlevels in mesocyclonic storms and in particular in combination with the occurrence of a tornado (e.g., Wurman 2002; Bluestein 2007).

In some cases, observed hook echoes at, say, 1-3 km AGL display a sharp bend or "kink" (e.g., French et al. 2008, their Fig. 6g). As Letzmann's vortex model is not limited to winds near the surface, we may apply his concepts also to parts of the vortex higher up. Accordingly, the velocity fields in Fig. 12 suggest that these "kinky hooks" might result from the interaction between the tornado vortex and the mesocyclone. If so, the interior velocity maximum would belong to the tornado (mesocyclone), while the exterior velocity peak would be produced by the larger mesocyclone aloft. A detailed analysis of this effect using the model presented here is the subject of ongoing work.

\section{Conclusions}

The method presented here allows reconstruction of near-surface tornado wind fields from the analysis of actual forest damage patterns. By simulating the observed tree damage patterns, the intensity and relevant parameters characterizing the wind field can be obtained:
- The analytical tornado model of Letzmann (1923) depending on the parameters $G_{\max }, \alpha$, and $v_{\text {trans }}$ is perfectly suited to determine these parameters based on the forest damage patterns.

- If the tornado translation speed is known, the damage pattern completely determines the wind field and its intensity in the Letzmann model; that is, intensity can be inferred without knowledge of the actual tree stand parameters.

- The translation speed of the tornado-producing thunderstorm as determined, for example, from radar observations may be used as a valid proxy to the tornado translation speed.

- Through consecutive simulations using varying $G_{\max }$, $\alpha$, and $R_{\max }$ to fit the observed damage patterns, the near-surface tornado wind fields and the location of the centerline of the tornado track can be determined.

- The convergence and divergence lines first mentioned by Letzmann (1923) could be verified in the observed tree damage patterns, leading to a better damage classification.

- Analyses of the observed tree damage patterns of the Milosovice and Castellcir tornadoes led to a verification of their $\mathrm{F} 3$ and $\mathrm{F} 2$ ratings, respectively.

- The distribution of the F scale along and across the path reveals the areal percentage of maximum intensity and may be used in risk models.

- As compared with the determination of tornado intensities from damage, the maximum velocity of the Milosovice tornado was determinable with an uncertainty of only $\pm 6.0 \mathrm{~m} \mathrm{~s}^{-1}$, less than one half-level of the $\mathrm{F}$ scale, while in general, the relation $\Delta v_{\max }=$ $\left(G_{\max }+1\right) \Delta v_{\text {trans }}$ holds. This point is encouraging, given the fact that the main objective of the method was to reconstruct the tornado near-surface wind field structure.

Aside from the ongoing work applying our model to vortex levels aloft with a double wind maximum to explain the often-observed "kinky hooks," a fruitful option for future work would be to extend the Letzmann formalism to three dimensions as already outlined by Letzmann (1923) and to dynamically simulate the lowest, say, $100 \mathrm{~m}$ of an advancing tornado.

Acknowledgments. The authors are grateful to Martin Setvák, Czech Hydrometeorological Institute, for providing radar images and aerial photographs of the Milosovice tornado and to Joan Bech, Meteorological Service of Catalonia, for information on his Castellcir tornado analysis. Leigh Orf, Central Michigan University, kindly shared data from his downburst wind field simulation and Michael Kasperski, Ruhr-Universität 
Bochum, provided the Gaussian distribution of the modulus of rupture. Frank Holzäpfel commented on a draft of this paper. Our three anonymous reviewers provided insightful and inspiring comments. This work was partly funded by the German Ministry for Education and Research BMBF under Contract 01LS05125 in the project RegioExAKT [Regionales Risiko konvektiver Extremwetterereignisse: Anwenderorientierte Konzepte zur Trendbewertung und -anpassung (Regional risk of convective extreme weather events: User-oriented concepts for trend assessment and adaptation)].

\section{REFERENCES}

Aran, M., J. Amaro, J. Arús, J. Bech, F. Figuerola, M. Gayà, and E. Vilaclara, 2009: Synoptic and mesoscale diagnosis of a tornado event in Castellcir, Catalonia, on 18th October 2006. Atmos. Res., 93 (1-3), 147-160.

Baldwin, V. C., 1987: Green and dry-weight equations for aboveground components of planted Loblolly pines in the West Gulf region. South. J. Appl. For., 11, 212-218.

Bech, J., M. Gayà, M. Aran, F. Figuerola, J. Amaro, and J. Arús, 2007: Tornado damage analysis of a forest area using site survey observations, radar data and a simple analytical vortex model. Preprints, Fourth European Conf. on Severe Storms, Trieste, Italy, ICTP/ARPA FVG, 2 pp. [Available online at www.essl. org/ECSS/2007/abs/02-Case-study/1177974332.bech-1-sec02. oral.pdf.]

,,,,---- , and,- 2009: Tornado damage analysis of a forest area using site survey observations, radar data and a simple vortex model. Atmos. Res., 93 (1-3), $118-130$

Beck, V., 2008: Near-surface tornado wind field reconstruction from forest damage. Diploma thesis, TU München, $162 \mathrm{pp}$. [Available online at http://www.essl.org/pdf/Beck2008.pdf.]

— wind fields from forest damage. Preprints, Fifth European Conf. on Severe Storms, Landshut, Germany, ESSL, 2 pp. [Available online at http://www.essl.org/ECSS/2009/preprints/ O11-05-beck.pdf.]

$\longrightarrow$ - - , and R. Sausen, 2008: Determination of tornado intensity from forest damage. Preprints, 24th Conf. on Severe Local Storms, Savannah, GA, Amer. Meteor. Soc., 8B.2. [Available online at http://ams.confex.com/ams/pdfpapers/ 141809.pdf.]

Bluestein, H. B., 2007: Mobile Doppler radar observations of tornadoes. Preprints, Fourth European Conf. on Severe Storms, Trieste, Italy, ICTP/ARPA FVG, 2 pp. [Available online at http://www.essl.org/ECSS/2007/abs/05-Radars/bluestein1177432754.pdf.]

Blumenfeld, K. A., 2008: Comments on "Low-level winds in tornadoes and potential catastrophic tornado impacts in urban areas." Bull. Amer. Meteor. Soc., 89, 1578-1579.

Brooks, H. E., 2004: On the relationship of tornado path length and width to intensity. Wea. Forecasting, 19, 310-319.

, and C. A. Doswell III, 2001: Some aspects of the international climatology of tornadoes by damage classification. Atmos. Res., 56, 191-201.

$\ldots, \ldots$, and D. Sutter, 2008: Comments on "Low-level winds in tornadoes and potential catastrophic tornado impacts in urban areas." Bull. Amer. Meteor. Soc., 89, 87-90.
Budney, L. J., 1965: Unique damage patterns caused by a tornado in dense woodlands. Weatherwise, 18, 74-77, 86.

Davies-Jones, R. P., 1986: Tornado dynamics. Thunderstorm Morphology and Dynamics, 2nd ed. E. Kessler, Ed., University of Oklahoma Press, 197-236.

Doswell, C. A., III, Ed., 2001: Severe Convective Storms. Meteor. Monogr., No. 28, Amer. Meteor. Soc., 561 pp.

— tornado climatology. Mon. Wea. Rev., 116, 495-501.

, H. E. Brooks, and N. Dotzek, 2009: On the implementation of the enhanced Fujita scale in the USA. Atmos. Res., 93 (1-3), $554-563$.

Dotzek, N., 2007: Derivation of physically motivated wind speed scales. Preprints, Fourth European Conf. on Severe Storms, Trieste, Italy, ICTP/ARPA FVG, 2 pp. [Available online at http://www.essl.org/ECSS/2007/abs/01-Theory/dotzek-2-sec01. oral-new.pdf.]

, 2009: Derivation of physically motivated wind speed scales. Atmos. Res., 93 (1-3), 564-574.

— , and K. Friedrich, 2009: Downburst-producing thunderstorms in southern Germany: Radar analysis and predictability. Atmos. Res., 93 (1-3), 457-473.

- G. Berz, E. Rauch, and R. E. Peterson, 2000: Die Bedeutung von Johannes P. Letzmanns "Richtlinien zur Erforschung von Tromben, Tornados, Wasserhosen und Kleintromben" für die heutige Tornadoforschung (The relevance of Johannes P. Letzmann's "Guidelines for research on tornadoes, waterspouts, and whirlwinds" for contemporary tornado research). Meteor. Z., 9, 165-174.

_ _ J. Grieser, and H. E. Brooks, 2003: Statistical modeling of tornado intensity distributions. Atmos. Res., 67-68, 163-187.

— M. V. Kurgansky, J. Grieser, B. Feuerstein, and P. Névir, 2005: Observational evidence for exponential tornado intensity distributions over specific kinetic energy. Geophys. Res. Lett., 32, L24813, doi:10.1029/2005GL024583.

_, P. Lang, M. Hagen, T. Fehr, and W. Hellmiss, 2007: Doppler radar observation, CG lightning activity, and aerial survey of a multiple downburst in southern Germany on 23 March 2001. Atmos. Res., 83, 519-533.

— R. E. Peterson, B. Feuerstein, and M. Hubrig, 2008: Comments on "A simple model for simulating tornado damage in forests." J. Appl. Meteor. Climatol., 47, 726-731.

_, P. Groenemeijer, B. Feuerstein, and A. M. Holzer, 2009: Overview of ESSL's severe convective storms research using the European Severe Weather Database ESWD. Atmos. Res., 93 (1-3), 575-586.

Euteneuer, G. A., 1970: Aerodynamische Betrachtungen im Pforzheimer Wirbelsturm vom 10. Juli 1968 (Aerodynamic considerations for the Pforzheim tornado of 10 July 1968). Arch. Meteor. Geophys. Bioklimatol., A19, 355-371.

Forbes, G. S., and R. M. Wakimoto, 1983: A concentrated outbreak of tornadoes, downbursts and microbursts, and implications regarding vortex classification. Mon. Wea. Rev., 111, 220-235.

French, M. M., H. B. Bluestein, D. C. Dowell, L. J. Wicker, M. R. Kramar, and A. L. Pazmany, 2008: High-resolution, mobile Doppler radar observations of cyclic mesocyclogenesis in a supercell. Mon. Wea. Rev., 136, 4997-5016.

Fujita, T. T., 1981: Tornadoes and downbursts in the context of generalized planetary scales. J. Atmos. Sci., 38, 1511-1534. , 1989: The Teton-Yellowstone tornado of 21 July 1987. Mon. Wea. Rev., 117, 1913-1940. 
Gardiner, B. A., G. R. Stacey, R. E. Belcher, and C. J. Wood, 1997: Field and wind tunnel assessments of the implication of respacing and thinning for tree stability. Forestry, 70, 233-252.

_, S. Hale, A. Wellpott, and B. Nicoll, 2009: The development of a wind risk model for irregular stands. Proc. Second Int. Conf. on Wind Effects on Trees, Freiburg, Germany, AlbertLudwigs-University of Freiburg, 127-133. [Available online at http://www.wind2009.uni-freiburg.de/Abstractband_komplett_ 7.10.2009.pdf.]

Hall, F., and R. D. Brewer, 1959: A sequence of tornado damage patterns. Mon. Wea. Rev., 87, 207-216.

Holland, A. P., A. J. Riordan, and E. C. Franklin, 2006: A simple model for simulating tornado damage in forests. J. Appl. Meteor. Climatol., 45, 1597-1611.

Hubrig, M., 2004: Analyse von Tornado- und DownburstWindschäden an Bäumen (Analysis of tornado and downburst wind damage to trees). Forst Holz, 59, 78-84.

Kanak, K. M., 2005: Numerical simulation of dust devil-scale vortices. Quart. J. Roy. Meteor. Soc., 131, 1271-1292.

Knupp, K. R., 2000: Narrow streaks of "straight-line" wind damage: Do tornadoes or microbursts produce them? Preprints, 20th Conf. on Severe Local Storms, Orlando, FL, Amer. Meteor. Soc., 644-645.

Lee, W.-C., and J. Wurman, 2005: Diagnosed three-dimensional axisymmetric structure of the Mulhall tornado on 3 May 1999. J. Atmos. Sci., 62, 2373-2393.

Letzmann, J. P., 1923: Das Bewegungsfeld im Fuß einer fortschreitenden Wind- oder Wasserhose (The flow field at the base of an advancing tornado). Ph.D. thesis, University Helsingfors, Acta et Commentationes Universitatis Dorpatensis AVI.3, 136 pp. [Available online at http://essl.org/pdf/ Letzmann1923/Letzmann1923.pdf.]

__, 1925: Fortschreitende Luftwirbel (Advancing air vortices). Meteor. Z., 42, 41-52.

, 1939: Richtlinien zur Erforschung von Tromben, Tornados, Wasserhosen und Kleintromben (Guidelines for research on tornadoes, waterspouts, and whirlwinds). Klimatologische Kommission, IMO Publ. 38, Secretariat de l'Organisation Météorologique Internationale, Ed., Edouard Ijdo, 91-110. [Available online at http://essl.org/pdf/Letzmann1939/ Letzmann1939.pdf.]

Lewellen, D. C., and W. S. Lewellen, 2007: Near-surface intensification of tornado vortices. J. Atmos. Sci., 64, 2176-2194.

_ B. Bong, and W. S. Lewellen, 2008: Effects of finescale debris on near-surface tornado dynamics. J. Atmos. Sci., 65, 3247-3262.

Müldner, W., 1950: Die Windbruchschäden des 22.7.1948 im Reichswald bei Nürnberg, ein Beispiel für ein Wirbelfeld als
Teilerscheinung einer Böenfront (The wind damage of 22 July 1948 in the Reichswald forest near Nuremberg, an example of a vortex field as a local phenomenon of a gust front). Ber. Dtsch. Wetterdienstes, 19, 3-29.

Peltola, H., and S. Kellomäki, 1993: A mechanistic model for calculating windthrow and stem breakage of Scots pines at stand edge. Silva Fenn., 27, 99-111.

— S S. Dupont, V.-P. Ikonen, H. Väisänen, A. Venälainen, and S. Kellomäki, 2009: The development of a wind risk model for irregular stands. Proc. Second Int. Conf. on Wind Effects on Trees, Freiburg, Germany, Albert-Ludwigs-University of Freiburg, 189-194. [Available online at http://www.wind2009. uni-freiburg.de/Abstractband_komplett_7.10.2009.pdf.]

Peterson, C. J., 2003: Factors influencing treefall risk in tornadoes in natural forests. Preprints, Symp. on the F-Scale and SevereWeather Damage Assessment, Long Beach, CA, Amer. Meteor. Soc., 3.1. [Available online at http://ams.confex.com/ams/ pdfpapers/53292.pdf.]

Peterson, R. E., 1992a: Johannes Letzmann: A pioneer in the study of tornadoes. Wea. Forecasting, 7, 166-184.

_ 1992b: Letzmann's and Koschmieder's "Guidelines for research on funnels, tornadoes, waterspouts and whirlwinds." Bull. Amer. Meteor. Soc., 73, 597-611.

Potter, S., 2007: Fine-tuning Fujita. Weatherwise, 60 (2), 64-71.

Prandtl, L., 1925: Berichte über Untersuchungen zur ausgebildeten Turbulenz (Reports on investigations on fully developed turbulence). Z. Angew. Math. Mech., 5, 136-139.

Rossmann, F., 1959: Über Baumzerstörung durch Tromben, besonders durch Verdrehen der Bäume (On tree destruction by tornadoes, especially by torsion of the trees). Meteor. Rundsch., 12 (5), 161-162.

Wegener, A., 1917: Wind- und Wasserhosen in Europa (Tornadoes in Europe). Verlag Friedrich Vieweg und Sohn, 301 pp. [Available online at http://www.essl.org/pdf/Wegener1917/.]

Wurman, J., 2002: The multiple-vortex structure of a tornado. Wea. Forecasting, 17, 473-505.

— , and C. R. Alexander, 2005: The 30 May 1998 Spencer, South Dakota, storm. Part II: Comparison of observed damage and radar-derived winds in the tornadoes. Mon. Wea. Rev., 133, 97-119.

$\longrightarrow,-$ - P. Robinson, and Y. Richardson, 2007: Low-level winds in tornadoes and potential catastrophic tornado impacts in urban areas. Bull. Amer. Meteor. Soc., 88, 31-46.

,,--- , and $\longrightarrow, 2008$ a: Reply. Bull. Amer. Meteor. Soc., 89, 90-94.

89, 1580-1581. 